\title{
Grain-boundary modelling of hydrogen assisted intergranular stress corrosion cracking
}

\author{
Ivano Benedetti ${ }^{\mathrm{a}, *}$, Vincenzo Gulizzi ${ }^{\mathrm{a}}$, Alberto Milazzo ${ }^{\mathrm{a}}$ \\ ${ }^{a}$ Department of Civil, Environmental, Aerospace, and Materials Engineering - DICAM, \\ Università degli Studi di Palermo, Viale delle Scienze, Edificio 8, \\ Palermo, 90128, Italy.
}

\begin{abstract}
A novel hybrid strategy for modelling intergranular hydrogen embrittlement in polycrystalline microstructures is proposed. The technique is based on a grain-boundary integral representation of the polycrystalline micro-mechanics, numerically solved by the boundary element method, coupled with an explicit finite element model of the intergranular hydrogen diffusion. The intergranular interaction between contiguous grains in the aggregate is modelled through extrinsic cohesive-frictional traction-separation laws, whose parameters depend on the concentration of intergranular hydrogen, which diffuses over the interface according to the Fick's second law, inducing the weakening of the interface itself. The model couples the advantages of the boundary element representation of the polycrystalline micro-mechanics, namely the reduction of the mechanical degrees of freedom, with the generality of the finite element modelling of the diffusion process, which in principle allows the straightforward coupling of the interfacial effective diffusivity with other local mechanical parameters, e.g. the interfacial damage or displacement opening. Several numerical tests complete the study, showing the potential of the proposed technique.
\end{abstract}

Keywords: Polycrystalline materials, Stress corrosion cracking, Hydrogen embrittlement, Micro-mechanics, Boundary element method

\section{Introduction}

Stress Corrosion Cracking (SCC) is a specific case of environmentally assisted cracking in which the interaction between a definite susceptible material, a definite aggressive environment and sus-

\footnotetext{
* Corresponding author

Email address: ivano.benedetti@unipa.it (Ivano Benedetti)
} 
tained local stresses may induce failures at load levels lower than the nominal design loads. SCC may trigger structural failures without early visible or detectable deformation and it is then recognised as a common issue for engineering applications in the oil, off-shore, nuclear and aviation industries. The experimental characterisation of the behaviour of mechanical structures in aggressive environments is generally a long and expensive process, further complicated by the need to assess different operating conditions. For such reasons, there is relevant interest in the development of mathematical and computational models for the assessment of structures subjected to SCC conditions [1, 2, 3].

The occurrence of SCC is induced by complex nano/microscopic mechanic-chemical interactions between susceptible grain materials, grain-boundary segregation of impurities [4, 5] and aggressive environments, in which the chemical kinetics and material deformation processes, induced by local stresses, are mutually enhanced. Due to the complex and system-specific nature of such interactions, there is shared awareness that a universal explanation of environmental assisted cracking may not be possible.

However, for structures operating in hydrogen-rich environments, common for example in marine and nuclear engineering, one of the fundamental and most studied SCC mechanisms is the material embrittlement caused by the diffusion of environmental hydrogen within the material itself, known as Hydrogen Assisted Stress Corrosion Cracking (HASCC) or Hydrogen Embrittlement (HE). Several HE mechanisms have been suggested in the literature [6, 7, 8, such as hydrogen enhanced decohesion (HEDE), hydrogen enhanced localised plasticity (HELP), absorption induced dislocation emission (AIDE) and delayed hydride cracking (DHC). The picture is further complicated by the dependence of the transport of hydrogen within the crystal lattice on various factors such as temperature, pressure, microstructure, hydrostatic stress, plastic strain.

Several studies have focused on modelling the effects of hydrogen embrittlement on engineering structures. A popular methodology is based on the use of cohesive zone modelling within a finite element framework 11. In this approach, the cohesive laws, namely the cohesive strength and critical separation, and then ultimately the cohesive energy, are modified to take into account the local concentration of hydrogen, which acts so to degrade the interface strength, thus reducing the cohesive energy. The relationship between material degradation and hydrogen concentration is supported by first principles calculations [9, 10, as shown in Ref. 11], where a quantum mechanics informed cohesive law for hydrogen embrittlement modelling is proposed. In Ref. [2] the diffusion 
of hydrogen within a compact test specimen under mode I loading is analytically solved and the hydrogen concentration profile is in turn used to accordingly degrade the cohesive law used in front of the specimen notch, thus triggering the crack propagation. In Ref. [3], a hydrostatic stress dependent model for hydrogen diffusion is adopted and solved through a finite difference scheme and subsequently coupled with a cohesive finite element model to study the effect on the propagation

40 of annular cracks in round specimens; the effect of the inclusion of the hydrostatic terms is also critically assessed.

While the above models focus on the component scale, some SCC models explicitly taking into account the metal polycrystalline microstructure have been developed [12, 13, also motivated by the experimental observation of intergranular stress corrosion cracking [14, 15, 16]. Musienko and Cailletaud [17] have developed a grain-scale model for inter/trans-granular iodine-assisted stress corrosion cracking (IASCC) in Zircaloy tubes used in nuclear power plants, based on the explicit modelling of the fully three-dimensional diffusion process. Kamaya and Itakura proposed a threedimensional Voronoi-based continuum damage mechanics model for intergranular SCC [18]. A multiscale model for hydrogen assisted SCC was developed by Rimoli and Ortiz [19], using the explicit representation of an idealised micro-morphology and segregation-dependent cohesive laws based on Ref.[11].

In the present study, an original grain-boundary formulation for hydrogen assisted intergranular stress corrosion cracking is proposed. The model is based on a boundary integral framework for the analysis of polycrystalline materials $20,21,22$ and explicitly models the hydrogen diffusion at 55 the grain boundaries through Fick's second law. The degradation of the intergranular interfaces, due to hydrogen diffusion, is captured by degrading the cohesive strength of the traction-separation laws employed between adjacent grains; the effect of hydrogen concentration is reflected in the degradation of cohesive energy. From the numerical point of view, the proposed formulation is hybrid, as a boundary integral model is used to represent the mechanics of bulk grains, while a finite element scheme is employed to capture the diffusion at the grain boundaries: this hybrid strategy retains the advantages of the grain-boundary model and ensures simplicity and generality in the modelling of the diffusion process.

The outline of the paper is as follows. The key elements of the formulation, i.e. the mechanical boundary integral formulation, the cohesive laws and the model for the diffusion process, are given in Section 2. The numerical discretisation and the evolution algorithms are addressed in Section 
3. Section 4 reports the results of the performing computations, highlighting the capability of the method. Some observations about further research are discussed in Section 5 , before the concluding remarks.

\section{Grain-scale hybrid formulation}

The proposed grain-scale formulation for hydrogen assisted stress corrosion cracking is built starting from a Voronoi representation of the polycrystalline aggregate 22]: each grain is represented as a Voronoi convex polygon bounded by flat convex polygonal faces, which provides a first-order approximation of real polycrystalline microstructures and offers definite computational advantages (related to the use of flat surfaces), see Fig. (1). The tessellations in this study have been built by using either Voro++ (http://math.lbl.gov/voro++/) 23] or Neper (http://neper.sourceforge.net) 24].

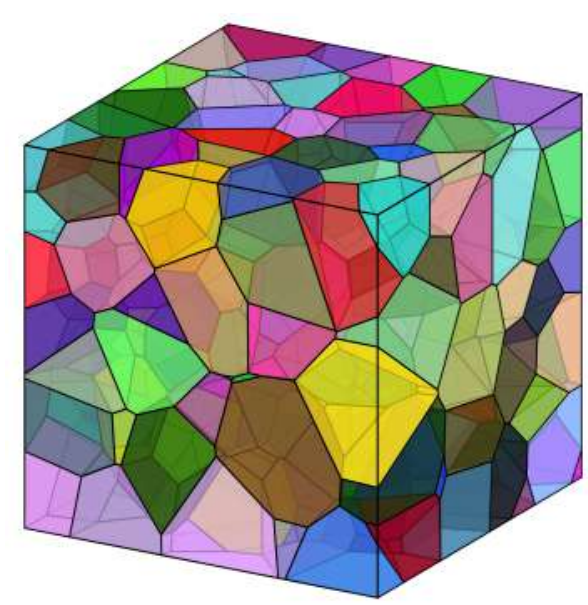

(a)

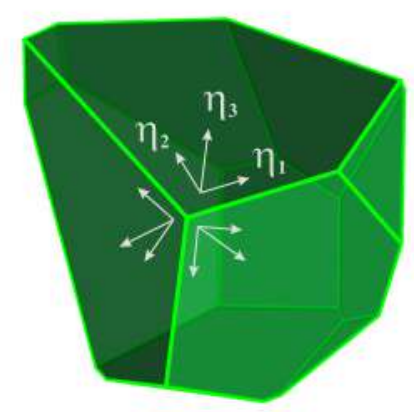

(b)

Figure 1: Example of artificial polycrystalline morphology: a) Voronoi tessellation with 200 grains; $b$ ) Voronoi grain with local reference systems set on different faces (note that the local reference systems are not generally aligned with grain faces edges).

The formulation is built by coupling: $a$ ) a grain-boundary integral representation of the mechanics of the bulk grains; $b$ ) a suitable set of intergranular interface conditions, including cohesive traction-separation laws embodying irreversible damage and hydrogen-segregation-dependent cohesive energy; c) a grain-boundary model for hydrogen diffusion, with a definition of effective 
diffusivity phenomenologically accounting for the complex processes related to the hydrogen diffusion.

For the sake of clarity, it is worth stressing that the primary variables of the formulation are grain-boundary displacements and tractions, so that these fields can be directly used to enforce the necessary interface equations.

\subsection{Bulk grains integral modelling}

The polycrystalline micro-morphology is modelled employing a multi-region boundary integral formulation in which a generic grain $g$ within the aggregate is considered as a linear elastic anisotropic domain, with a specific crystallographic orientation in the three-dimensional space. The grain $g$ is bounded by the grain boundary $S^{g}$, which is formed, as already mentioned, by the union of flat convex polygonal faces. The displacement boundary integral equation [25, 26]

$$
\widetilde{c}_{i j}^{g}(\boldsymbol{\xi}) \widetilde{u}_{j}^{g}(\boldsymbol{\xi})+f_{S^{g}} \widetilde{T}_{i j}^{g}(\boldsymbol{\xi}, \boldsymbol{\eta}) \widetilde{u}_{j}^{g}(\boldsymbol{\eta}) \mathrm{d} S(\boldsymbol{\eta})=\int_{S^{g}} \widetilde{U}_{i j}^{g}(\boldsymbol{\xi}, \boldsymbol{\eta}) \widetilde{t}_{j}^{g}(\boldsymbol{\eta}) \mathrm{d} S(\boldsymbol{\eta})
$$

can be written for each grain $g$ of the aggregate. In Eq.(1), $\boldsymbol{\xi}$ is the generic collocation point, at which the boundary integral equation is evaluated, while $\boldsymbol{\eta}$ is the generic integration point, which runs over the boundary of the grain $S^{g} ; \widetilde{u}_{j}^{g}$ and $\widetilde{t}_{j}^{g}$ are components of the displacement and traction fields over $S^{g}$, with the symbol denoting quantities calculated over a specific face of the grain's boundary, i.e. with respect to the grain-face local reference system, which varies as the integration point runs over the grain's boundary, see e.g. Refs. 20, 21] and Fig. $[1] ; \widetilde{U}_{i j}^{g}(\boldsymbol{\xi}, \boldsymbol{\eta})$ and $\widetilde{T}_{i j}^{g}(\boldsymbol{\xi}, \boldsymbol{\eta})$ are obtained by suitably rotating the components of the 3D anisotropic fundamental solutions of the grain material, which are computed as explained in Ref. 27] and recalled in Appendix A $\widetilde{c}_{i j}^{g}(\boldsymbol{\xi})$ are

the free terms arising from the boundary limiting procedure and depending on the smoothness of the boundary $S^{g}$ [25, 26]. In summary, Eq.(1) allows to express the displacements at the collocation points in terms of the displacements and tractions over the grain-boundary.

\section{2. intergranular interfaces modelling}

To model the polycrystalline aggregate, the equations written for the individual bulk grains must be coupled through suitable interface equations. A region on an intergranular interface may be pristine, damaged or failed. Let us consider a couple of homologous intergranular points $P^{a}$ and $P^{b}$, belonging to the two contiguous grains $a$ and $b$; if damage is not yet initiated under the given 
local loading conditions, then no relative displacements are allowed between them and interface continuity requires

$$
\left\{\begin{array}{lrl}
\text { displacements continuity } & \delta \tilde{u}_{i}^{a b}=-\left(\tilde{u}_{i}^{a}+\tilde{u}_{i}^{b}\right)=0 & i=1,2,3 . \\
\text { tractions equilibrium } & \tilde{t}_{i}^{a}=\tilde{t}_{i}^{b}
\end{array}\right.
$$

In each solution step, the displacement and traction fields at the grain boundaries are completely resolved; the local tractions can then be employed to check the threshold condition

$$
t_{e}=\left[\left\langle\tilde{t}_{n}\right\rangle^{2}+\left(\frac{\beta}{\alpha} \tilde{t}_{s}\right)^{2}\right]^{\frac{1}{2}} \geq T_{M}(\phi)
$$

where $t_{e}$ is an effective traction expressed in terms of the normal traction component $\tilde{t}_{n}=\tilde{t}_{3}$ and

tangential traction component $\tilde{t}_{s}=\sqrt{\tilde{t}_{1}^{2}+\tilde{t}_{2}^{2}}$ and $T_{M}(\phi)$ is a cohesive strength depending on the hydrogen relative concentration $\phi$, defined in Section 2.3 the coefficients $\alpha$ and $\beta$ are cohesive constants, see e.g. 28, 21, while $\langle\cdot\rangle$ denotes the Macauley's brackets.

The degradation of the cohesive strength with the hydrogen concentration is expressed in this work by the relation

$$
T_{M}(\phi)=(1-\gamma \phi) T_{0},
$$

where $T_{0}$ is the cohesive strength of the interface without the presence of diffused hydrogen, $\gamma$ is a non-dimensional parameter expressing the interface degradation accompanying the diffusion of hydrogen [1, 2, 3].

When the threshold condition in Eq.(3) is fulfilled, then damage initiates and continuity does not hold anymore; the displacement continuity relationships in Eq.22) are then replaced by the extrinsic mixed-mode traction-separation laws with irreversible damage and hydrogen-concentrationdependent cohesive energy

$$
\left[\begin{array}{c}
\tilde{t}_{s} \\
\tilde{t}_{n}
\end{array}\right]=T_{M}(\phi) \frac{1-d^{*}}{d^{*}}\left[\begin{array}{cc}
\frac{\alpha}{\delta u_{s}^{c r}} & 0 \\
0 & \frac{1}{\delta u_{n}^{c r}}
\end{array}\right]\left[\begin{array}{c}
\delta \tilde{u}_{s} \\
\delta \tilde{u}_{n}
\end{array}\right]
$$

where

$$
d^{*}=\max _{\mathscr{H}_{d}}\{d\} \in[0,1] \quad \text { with } \quad d=\left[\left\langle\frac{\delta \tilde{u}_{n}}{\delta u_{n}^{c r}}\right\rangle^{2}+\beta^{2}\left(\frac{\delta \tilde{u}_{s}}{\delta u_{s}^{c r}}\right)^{2}\right]^{\frac{1}{2}},
$$

is the damage parameter, which is always given by the maximum value reached by the effective displacement $d$ during the loading history $\mathscr{H}_{d}$ of the considered interface pair. In Eq. (6), $\delta \tilde{u}_{n}=\delta \tilde{u}_{3}$ 
and $\delta \tilde{u}_{s}=\sqrt{\delta \tilde{u}_{1}^{2}+\delta \tilde{u}_{2}^{2}}$ are the normal and tangential displacement jumps and $\delta u_{n}^{c r}$ and $\delta u_{s}^{c r}$ are the critical jumps in pure mode I and II, respectively. It is worth noting that Eqs. (4) 5 ) correspond to the assumption that the hydrogen absorption affects simultaneously both mode I and mode II, with the same percentage degradation with respect to the original values of strength.

Upon failure, when the critical condition $d^{*}=1$ is reached, the cohesive laws no longer apply and frictional contact mechanics is introduced to model the micro-cracks, whose surfaces may separate, slip or stick.

\subsection{Hydrogen diffusion modelling}

In this work, only the intergranular diffusion of hydrogen is considered, in agreement with the assumptions also adopted in Ref.[19], which apply to large grains at room temperature.

Considering the generic intergranular interface $\mathscr{I}^{a b}$ between the two contiguous grains $a$ and $b$, which in the Voronoi representation of the polycrystalline microstructure is a flat convex polygon bounded by straight segments, hydrogen diffusion is modelled introducing a relative concentration function $\phi(\boldsymbol{\eta}, t)=C(\boldsymbol{\eta}, t) / C_{\text {sat }}$, where $\boldsymbol{\eta}=\left(\eta_{1}, \eta_{2}\right)$ denotes the coordinates of a generic interfacial point $P \in \mathscr{I}^{a b}$ expressed in a local reference system, see Fig. $1 \mathrm{~b}, t$ represents the time variable and $C(\boldsymbol{\eta}, t)$ and $C_{\text {sat }}$ are the local absolute and saturation values of hydrogen concentration respectively. It is worth noting that $C_{s a t}$ is a material parameter that could be experimentally determined; however, the knowledge of its specific value does not play any role in the present diffusion formulation, which is expressed in terms of relative concentration, as the truly relevant information is how much the strength of a generic intergranular interface is degraded at saturation, which is expressed by the non-dimensional material parameter $\gamma$ entering Eq.(4).

Hydrogen diffuses over each generic interface $\mathscr{I}^{a b}$ according to the two-dimensional second Fick's law

$$
\frac{\partial \phi}{\partial t}=\frac{\partial}{\partial \eta_{i}}\left(D \frac{\partial \phi}{\partial \eta_{i}}\right) \quad \forall \boldsymbol{\eta}=\left(\eta_{1}, \eta_{2}\right) \in \mathscr{I}^{a b}
$$

with the associated initial and boundary conditions

$$
\phi(\boldsymbol{\eta}, 0)=\bar{\phi}_{0} \quad \forall \boldsymbol{\eta} \in \mathscr{I}^{a b}, \quad \begin{cases}\phi(\boldsymbol{\eta}, t)=\bar{\phi}(t) & \forall \boldsymbol{\eta} \in \mathscr{C}_{\phi}^{a b}=\partial \mathscr{I}_{\phi}^{a b} \\ q(\boldsymbol{\eta}, t)=\bar{q}(t) & \forall \boldsymbol{\eta} \in \mathscr{C}_{q}^{a b}=\partial \mathscr{I}_{q}^{a b}\end{cases}
$$

where $q=-D n_{i}\left(\partial \phi / \partial \eta_{i}\right)$ and $D$ is the interface effective diffusivity accounting for the effects of the grain-boundary microstructural features [11. In general, the effective diffusivity is related to the 
local interface state, e.g. intergranular opening $\delta \tilde{\boldsymbol{u}}$ and damage state $d^{*}$, thus affecting the microfluidic penetration of the aggressive agents. The dependency of $D$ on the interface local opening and/or degradation status introduces a mechanic/chemical coupling in the formulation. However, as pointed out by Rimoli and Ortiz [19], the reliable determination of such dependency is a difficult task and it goes well beyond the scope of the present study, so that a constant value of effective diffusivity is assumed in the implemented formulation.

The definition of the boundary conditions of the problem, especially in the case of fully developed cracks within the microstructure, deserves some considerations. Depending on the nature of the aggressive environment, the development of cracks would induce the penetration of the aggressive agents within the microstructure itself, thus redefining the extension of the area affected by the external conditions, as the newly formed crack surfaces would get in contact with the environment 19. However, the inclusion of such effects in the present framework would require the explicit modelling of the material/environment interaction, with specific chemical kinetics considerations. These effects are neglected in this study and simple Dirichlet boundary conditions on the external walls of the polycrystalline aggregate are adopted. More specifically, the walls of the aggregate exposed to the environmental action are forced to values of relative hydrogen concentration varying from zero to one, corresponding to the saturation value, within a finite time $T_{\text {sat }}$, in order to avoid physical inconsistence of the diffusion process and ensure numerical stability. Further comments about this aspect are given at the beginning of Section 4 .

\section{Numerical discretisation and algorithms}

The numerical solution of the HASCC problem is obtained by adopting a hybrid approach based both on the boundary element treatment of the integral equations used for polycrystalline mechanics and on the finite element discretisation of the intergranular diffusion equations.

\subsection{Boundary element discretisation of the bulk grains}

To solve numerically the polycrystalline problem, Eq.(1) is employed in the framework of the boundary element method for each grain $g$ of the aggregate, according to the following steps:

- The boundary of each grain is subdivided into a collection of non-overlapping quadrangular and triangular elements, following the meshing strategy developed in Ref.[22]; 
- The boundary displacement and traction fields are expressed in terms of suitable shape functions $\boldsymbol{N}_{e l}\left(\eta_{1}, \eta_{2}\right)$, defined over each element in a local 2D (surface) coordinate system $\left(\eta_{1}, \eta_{2}\right)$, and nodal values of boundary displacements $\widetilde{\boldsymbol{U}}^{g}$ and tractions $\widetilde{\boldsymbol{T}}^{g}$, expressed in a face-local reference system. After this discretisation procedure, a set of nodal points and boundary elements are associate to each grain;

- Eq.(1) is collocated at each node of the considered grain and it is numerically integrated, considering the explicit approximation of the boundary fields in terms of shape functions and nodal values, as detailed in Ref. 22 ]

In this way, a set of $3 \times N_{p}^{g}$ equations, where $N_{p}^{g}$ is the number of discretisation nodes associated with the grain $g$, is written in terms of nodal values of displacements and tractions for each node as

$$
\widetilde{\boldsymbol{H}}^{g} \cdot \widetilde{\boldsymbol{U}}^{g}=\widetilde{\boldsymbol{G}}^{g} \cdot \widetilde{\boldsymbol{T}}^{g}
$$

where $\widetilde{\boldsymbol{H}}^{g}$ and $\widetilde{\boldsymbol{G}}^{g}$ are the matrices stemming from the boundary integration of the kernels $\widetilde{T}_{i j}^{g}$ and $\widetilde{U}_{i j}^{g}$ respectively. During the numerical integration, care must be taken when integrating over the elements that contain, for a given collocation point, the collocation point itself. Such singular elements must be suitably treated [25, 26].

Enforcing displacement and traction boundary conditions on the faces of the grains lying on the loaded walls of the aggregate leads, for each grain, to the following system of equations

$$
\boldsymbol{A}^{g} \cdot \boldsymbol{X}^{g}=\boldsymbol{C}^{g} \cdot \boldsymbol{Y}^{g}
$$

where $\boldsymbol{X}^{g}$ collects the unknown values of grain-boundary displacements and tractions, $\boldsymbol{Y}^{g}$ collects prescribed values of boundary displacements and tractions, and $\boldsymbol{A}$ and $\boldsymbol{C}$ collect suitably related combinations of columns of the matrices $\widetilde{\boldsymbol{H}}^{g}$ and $\widetilde{\boldsymbol{G}}^{g}[26$.

Equation (10) is written for each grain of the aggregate; the enforcement of suitable intergranular conditions then leads to the system

$$
\left[\begin{array}{c}
\boldsymbol{A} \cdot \boldsymbol{X} \\
\boldsymbol{I}\left(\boldsymbol{X}, \boldsymbol{\phi}, \boldsymbol{d}^{*}\right)
\end{array}\right]=\left\{\begin{array}{c}
\boldsymbol{C} \cdot \boldsymbol{Y}(\lambda) \\
\mathbf{0}
\end{array}\right\}
$$


where

$$
\boldsymbol{A}=\left[\begin{array}{cccc}
\boldsymbol{A}^{1} & \mathbf{0} & \cdots & \mathbf{0} \\
\mathbf{0} & \boldsymbol{A}^{2} & \cdots & \mathbf{0} \\
\vdots & \vdots & \ddots & \vdots \\
\mathbf{0} & \mathbf{0} & \cdots & \boldsymbol{A}^{N_{g}}
\end{array}\right], \quad \boldsymbol{C}=\left[\begin{array}{cccc}
\boldsymbol{C}^{1} & \mathbf{0} & \cdots & \mathbf{0} \\
\mathbf{0} & C^{2} & \cdots & \mathbf{0} \\
\vdots & \vdots & \ddots & \vdots \\
\mathbf{0} & \mathbf{0} & \cdots & C^{N_{g}}
\end{array}\right]
$$

being $N_{g}$ the number of grains of the aggregate, $\boldsymbol{X}^{T}=\left\{\boldsymbol{X}^{1 T}, \ldots, \boldsymbol{X}^{N_{g} T}\right\}$ the vector collecting the unknown degrees of freedom, i.e. displacements and tractions of the overall aggregate, $\boldsymbol{Y}^{T}=\left\{\boldsymbol{Y}^{1 T}, \ldots, \boldsymbol{Y}^{N_{g} T}\right\}$ the vector collecting the prescribed values and $\lambda$ a suitable load factor. Eventually, $\boldsymbol{I}\left(\boldsymbol{X}, \boldsymbol{\phi}, \boldsymbol{d}^{*}\right)$ implements the interface conditions, which are in general function of the interface displacement jumps and traction fields, of the hydrogen concentration, whose nodal values are collected in the vector $\phi$, see Section 3.3 , and of the interface damage status, represented by the nodal damage variables collected in the vector $\boldsymbol{d}^{*}$.

\section{2. intergranular interface equations}

The interface equations account for the relationships between displacements and traction nodal values at the interface between contiguous grains. Denoting with $\mathscr{I}^{a b}$ the interface between two grains $a$ and $b$, the interface conditions can be generally expressed, $\forall \boldsymbol{\eta} \in \mathscr{I}^{a b}$, as

$$
\begin{aligned}
& \Psi_{i}^{a b}\left[\widetilde{u}_{j}^{a}(\boldsymbol{\eta}), \widetilde{u}_{j}^{b}(\boldsymbol{\eta}), \widetilde{t}_{j}^{a}(\boldsymbol{\eta}), \widetilde{t}_{j}^{b}(\boldsymbol{\eta}), \phi(\boldsymbol{\eta}), d^{*}(\boldsymbol{\eta})\right]=0 \quad i, j=1,2,3, \\
& \Phi_{i}^{a b}\left[\widetilde{t}_{j}^{a}(\boldsymbol{\eta}), \widetilde{t}_{j}^{b}(\boldsymbol{\eta})\right]=0
\end{aligned}
$$

where $\Psi_{i}^{a b}$ implements either continuity, cohesive or frictional contact laws, depending on the state $d^{*}(\boldsymbol{\eta})$ of the interface and on the local hydrogen concentration $\phi(\boldsymbol{\eta})$, while $\Phi_{i}^{a b}$ expresses traction equilibrium equations. Eqs.[12), written for all the interface node pairs, are collected in the matrix block $\boldsymbol{I}\left(\boldsymbol{X}, \boldsymbol{\phi}, \boldsymbol{d}^{*}\right)$ appearing in Eq. (11). Further details about intergranular micro-cracking modelling are given in Refs. 221, 22.

\subsection{Finite element discretisation for diffusion over the grains boundaries}

Hydrogen diffuses at the intergranular interfaces according to Fick's second law, as discussed in Section 2.3. For the numerical treatment of the diffusion equation, the generic interface $\mathscr{I}^{a b}$ is subdivided into a set on non-overlapping triangular and quadrangular elements, following the discretisation strategy presented in Ref.22] and outlined in Section 3.1. A standard finite element formulation can be obtained from a weighted residuals statement of the diffusion problem and then 
employing the approximation $\phi=N_{\alpha}(\boldsymbol{\xi}) \phi_{\alpha}(t)$ for both trial and test functions, where $N_{\alpha}(\boldsymbol{\xi})$ are suitable shape functions, specialised to triangular or quadrangular elements, and $\phi_{\alpha}(t)$ are relative concentration nodal values, with $\alpha=1, \ldots, N_{e}$, with $N_{e}$ expressing the number of element nodal points 29]. For the individual element $S_{k} \in \mathscr{I}^{a b}$, the following elemental algebraic equations are written

$$
M_{\alpha \beta}^{k} \dot{\phi}_{\beta}^{k}+K_{\alpha \beta}^{k} \phi_{\beta}^{k}+Q_{\alpha}^{k}+\bar{Q}_{\alpha}^{k}=0 \quad \alpha, \beta=1, \ldots, N_{e}
$$

where

$$
M_{\alpha \beta}^{k}=\int_{S_{k}} N_{\alpha}(\boldsymbol{\xi}) N_{\beta}(\boldsymbol{\xi}) d S, \quad K_{\alpha \beta}^{k}=\int_{S_{k}} D \frac{\partial N_{\alpha}(\boldsymbol{\xi})}{\partial \xi_{i}} \frac{\partial N_{\beta}(\boldsymbol{\xi})}{\partial \xi_{i}} d S
$$

and

$$
\bar{Q}_{\alpha}^{k}=\int_{\partial S_{k}^{\bar{q}}} N_{\alpha}(\boldsymbol{\xi}) \bar{q}(\boldsymbol{\xi}) d C
$$

while $Q_{\alpha}^{k}$ are the nodal flux sources (or sinks).

A diffusion finite element system for the whole polycrystalline morphology is obtained writing Eq. (13) for each interface element and following the standard finite element procedure, from the global re-numbering of nodes to the population of the global matrix, which eventually leads

$$
M \cdot \dot{\phi}+K \cdot \phi+Q=0
$$

where the vector $\phi$ collects the nodal values of relative concentrations and $\boldsymbol{Q}$ collects the nodal fluxes. In this work, only Dirichlet boundary conditions are considered for diffusion: for some nodes the relative concentration is assigned as a known function of time, while it is unknown for the remaining nodes. The boundary conditions then induce a partition of the system that, considering that no sources or sinks are located within the polycrystalline morphology, reads as

$$
\left[\begin{array}{ll}
M_{11} & M_{12} \\
M_{21} & M_{22}
\end{array}\right]\left[\begin{array}{l}
\dot{\phi}_{1} \\
\dot{\phi}_{2}
\end{array}\right]+\left[\begin{array}{ll}
\boldsymbol{K}_{11} & \boldsymbol{K}_{12} \\
\boldsymbol{K}_{21} & \boldsymbol{K}_{22}
\end{array}\right]\left[\begin{array}{l}
\phi_{1} \\
\boldsymbol{\phi}_{2}
\end{array}\right]+\left[\begin{array}{c}
\mathbf{0} \\
\boldsymbol{Q}_{2}
\end{array}\right]=\mathbf{0},
$$

where $\phi_{1}(t)$ collects the unknown nodal values of concentration and $\phi_{2}(t)$ collects the known nodal concentrations. The unknown $\phi_{1}$ are determined by solving the sub-system

$$
\boldsymbol{M}_{11} \cdot \dot{\boldsymbol{\phi}}_{1}+\boldsymbol{K}_{11} \cdot \boldsymbol{\phi}_{1}=-\boldsymbol{M}_{12} \cdot \dot{\boldsymbol{\phi}}_{2}-\boldsymbol{K}_{12} \cdot \boldsymbol{\phi}_{2}=\boldsymbol{R}(t)
$$

where $\phi_{2}$, and then $\boldsymbol{R}(t)$, is a linear function of time $\forall t \in\left[0, T_{\text {sat }}\right]$, while it remains constant $\forall t \geq T_{\text {sat }}$. 


\subsection{System coupling and incremental-iterative solution}

The hydrogen assisted stress corrosion cracking problem is numerically solved by simultaneously employing Eq. 18) and Eq. (11). The problem is formulated in incremental terms, looking for the solution of the diffusion/mechanical system at the time $t+\Delta t$ once the solution at the time $t$ is known. Since the effective diffusion at the intergranular interfaces is assumed as independent from the opening displacement jump and interface status, the diffusion evolution can be analytically determined from Eq.(18) as 30.

$$
\phi_{1}(t+\Delta t)=\exp \left\{-\boldsymbol{M}_{11}^{-1} \cdot \boldsymbol{K}_{11} \Delta t\right\} \cdot\left[\phi_{1}(t)-\phi_{1 p}(t)\right]+\phi_{1 p}(t+\Delta t),
$$

where $\exp \{\cdot\}$ denotes the exponential matrix and $\phi_{1 p}(t)$ is a particular solution of Eq. 18 that, due to the form of $\boldsymbol{R}(t)$, can be at most linear. In this work, the software package Expokit 31. (https://www.maths.uq.edu.au/expokit/) has been employed to compute the exponential matrix needed to solve the diffusion step. It is worth noting that, in principle, being it uncoupled from the micro-cracking evolution, the diffusion problem could be solved independently from time $t=0$ to the end of the time window of interest, without subdividing it into time steps $\Delta t_{k}$. However, the incremental algorithmic structure has been maintained to allow for more general solution cases.

Once the diffusion is solved, Eq. 11) can be rewritten as

$$
\left[\begin{array}{c}
\boldsymbol{A} \cdot \boldsymbol{X} \\
\boldsymbol{I}\left[\boldsymbol{X}, \boldsymbol{\phi}(t), \boldsymbol{d}^{*}\right]
\end{array}\right]=\left\{\begin{array}{c}
\boldsymbol{C} \cdot \boldsymbol{Y}[\lambda(t)] \\
\mathbf{0}
\end{array}\right\},
$$

which is solved employing the incremental-iterative technique developed in Refs. 21, 22, Due to the

highly sparse nature of system (20), PARDISO (http://www.pardiso-project.org/) 32, 33, 34] is used as solver. Enhanced computational efficiency, in the context of the boundary element method, could be achieved using fast iterative solvers in conjunction with special matrix representations, e.g. fast multipoles [35] or hierarchical matrices [36, 37, 38, for the solution of system 20

\section{Computational simulations}

The developed formulation has been implemented and tested to assess its performance and robustness and simulate the behaviour of polycrystalline aggregates under the combined action of a mechanical load and an aggressive environment. 
The simulations have been performed on CINECA's Galileo HPC infrastructure, employing 2.40 $\mathrm{GHz}$ nodes with 16 cores. Each node could access $128 \mathrm{~GB}$ of RAM and the maximum wall time issues were discussed and addressed in Ref.[12.

A parametric analysis has been performed to explore the effect of the effective diffusivity $D$ 


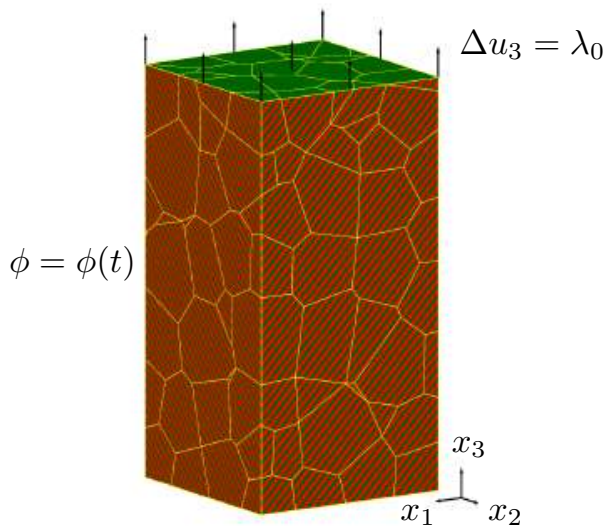

Figure 2: Schematic of the boundary conditions used in the relaxation tests: a 200-grain aggregate subjected to uniaxial constant strain along $x_{3}$ and exposed to an aggressive environment on the four lateral walls; the hydrogen concentration on the walls varies linearly from zero to the saturation value in a finite time $T_{\text {sat }}$.

and degradation parameter $\gamma$ on the relaxation curves; also the role of morphological changes and the influence of the pre-load level has been assessed and the results are summarised in Fig.(3). Fig.(3a) reports the time evolution of the macro-stress component $\Sigma_{33}$, obtained from the microstress volume average over all the grains of the aggregate, for four different 200-grain morphologies. The four different morphologies have been randomly generated using Neper [24; it is shown how, under the combined action of the pre-load and aggressive environment, they exhibit an analogous stress relaxation trend. In this sense, such morphologies can be assumed as representative of the material behaviour and subsequent tests are not biased by the morphological features of the specific considered aggregate. Such conclusion is based on the feeling matured in the use of the present framework also in previous studies 20, 21, 22, however, a systematic statistical investigation in this is sense has not been performed and it should be based on the analysis of a higher number of specimens.

Fig. (3b) explores the effect of the pre-load level on the stress relaxation of a selected aggregate, for a fixed value of the effective diffusivity and degradation parameter. It is seen that, as expected, the stress relaxation is more pronounced for aggregates subjected to higher initial strains. This is consequence of the fact that, although the aggressive environment acts in the same way on the aggregates subjected to different pre-loads, damage evolution is actually triggered only when the local intergranular stresses overcome the degraded activation threshold; low local stresses may not 
Table 1: Material properties of the simulated aggregate [12, 21].

\begin{tabular}{llll}
\hline \hline Domain & Property & Component & Value \\
\hline Bulk crystals (cubic) & Elastic constants [GPa] & $c_{11}, c_{22}, c_{33}$ & 204.6 \\
& & $c_{12}, c_{13}, c_{23}$ & 137.7 \\
& & $c_{44}, c_{55}, c_{66}$ & 126.2 \\
& Elastic anisotropic factor & $Z=2 c_{44} /\left(c_{11}-c_{12}\right)$ & 3.77 \\
\hline Grain boundaries & Interface strength [MPa] & $T_{0}$ & 205 \\
& & $\alpha$ & 1 \\
& Cohesive law constants [-] & $\beta$ & $\sqrt{2}$ \\
& & $\delta u_{n}^{c r}$ & $5.01 \cdot 10^{-2}$ \\
& Critical displacements jumps $[\mu \mathrm{m}]$ & $\delta u_{s}^{c r}$ & $\left(\beta^{2} / \alpha\right) \delta u_{n}^{c r}$ \\
\hline \hline
\end{tabular}

activate the damage evolution, despite the environmentally induced degradation of the intergranular cohesive strength. It is worth noting that specimens subjected to higher pre-loads may relax more and faster than specimens subjected to milder initial conditions, due to the higher levels of damage reached at the interfaces. In other words, in specimens subjected to high pre-loads, the initial diffusion induces the quick failure of the interfaces close to the lateral walls, which in turn induces a likely stress concentration on contiguous, but more internal interfaces. In such cases, the mechanical stress concentration, although triggered by the initial diffusion, may play a more important role than the diffusion itself with respect to the speed of the relaxation process. The effect of the degradation parameter $\gamma$ is reported in Fig. (3c): the same morphology relaxes more for higher values of $\gamma$; however, it is worth noting that the relaxation time is the same for all values of $\gamma$, as the speed of the relaxation is controlled by the diffusion process. Fig. (3d) shows the relaxation curves for different values of the effective diffusivity $D$. It clearly appears that all the curves attain the same relaxed value of macro-stress and that $D$ affects the speed of the relaxation process, by directly affecting the intergranular diffusion of the aggressive species.

Fig. (4) shows, for a fixed value of diffusivity $D=1 \mu \mathrm{m}^{2} / \mathrm{s}$, the contour plots, at different times, of the relative hydrogen concentration and damage levels, for different levels of pre-load $\lambda_{0}$ and degradation parameter $\gamma$. In Fig.4a, it is shown how, at $t=10 \mathrm{~s}$, the interfaces close to the external walls are already affected by hydrogen; this is due to the fact that, while the walls' 


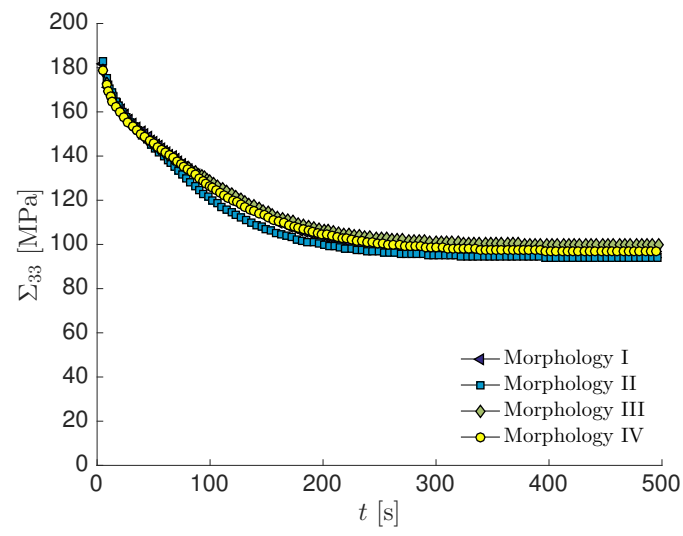

(a) $\lambda_{0}=4.0 \cdot 10^{-2} \mu \mathrm{m}, \gamma=0.5, D=1.0 \mu \mathrm{m}^{2} / \mathrm{s}$.

(b) $\gamma=0.5, D=1.0 \mu \mathrm{m}^{2} / \mathrm{s}$, Morphology I.
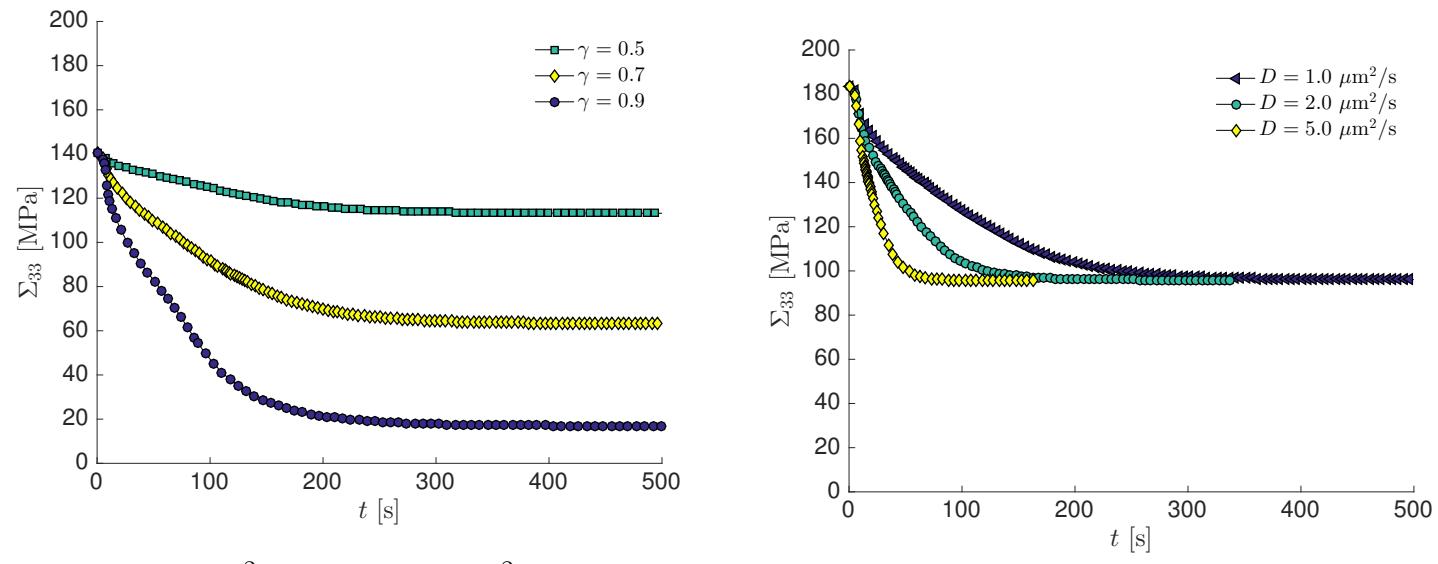

(c) $\lambda_{0}=3.0 \cdot 10^{-2} \mu \mathrm{m}, D=1.0 \mu \mathrm{m}^{2} / \mathrm{s}$, Morphology I.

(d) $\lambda_{0}=4.0 \cdot 10^{-2} \mu \mathrm{m}, \gamma=0.5$, Morphology I.

Figure 3: Stress relaxation curves $\Sigma_{33}(t)$ for 200-grain morphologies subjected to uniaxial pre-strain: $a$ ) behaviour of four different randomly generated morphologies; $b$ ) effect of pre-strain level; $c$ ) effect of the degradation parameter $\gamma ; d)$ effect of the diffusivity $D$. 
concentration evolves from zero to the saturation level in the interval $[0,10 \mathrm{~s}]$, the diffusion at the intergranular interfaces is not constrained, but follows the second Fick's law. Moreover, it is confirmed that, for a fixed value of $\gamma$, a higher pre-strain induces higher and more spread levels of damage; on the other hand, for fixed pre-strain, the higher the value of $\gamma$, the higher the average level of damage.

In the second series of tests, a 200-grain polycrystalline aggregate is simultaneously subjected to the action of an aggressive hydrogen environment and of a progressive uniaxial strain, applied by enforcing the displacement $\Delta u_{3}(t)=\lambda(t)=\dot{\lambda} t$ on the aggregate top surface, see Fig 5a. A parametric study is performed to assess the combined influence of the applied displacement rate and of the hydrogen diffusion for different values of effective diffusivity and degradation factor.

Figs. $5 \mathrm{~b}$. $5 \mathrm{c}$ ) show the macro stress-strain curves obtained under the action of a top-surface applied displacement rate of $\dot{\lambda}=3.36 \cdot 10^{-4} \mu \mathrm{m} / \mathrm{s}$ and $\dot{\lambda}=1.05 \cdot 10^{-4} \mu \mathrm{m} / \mathrm{s}$ (the specimen has an overall height of $\approx 56 \mu \mathrm{m}$ ) for different values of the diffusivity/degradation parameter. The degradation parameter has a direct effect on the peak values of macro stress-strain curves.

On the other hand, an interesting interplay between the applied displacement rate $\dot{\lambda}$ and the speed of the diffusion process, expressed by $D$, emerges from the comparison of the same two figures. This aspect is explicitly highlighted in Fig. (5d), where it is pointed out that, for sufficiently high values of $D$, the loading rate may not induce a difference between the stress-strain curves; this is due to the comparatively higher speed of the diffusion process with respect to the selected loading rates, so that the degradation threshold is met at the local interfaces at the same level of applied displacement. For smaller values of diffusivity, and then slower hydrogen diffusion, the effect of the loading rate becomes more apparent. It is stressed that this aspect does not depend on the value of $\gamma$, although different values of this parameter are selected in the figure for clarity of presentation.

Fig.(6) shows the damage evolution contour plots for the progressive loading tests with hydrogen environment acting over the four lateral faces of the aggregate. Different values of diffusivity, degradation parameter and loading rate are considered: in each case, the damage patterns appear quite spread, before the final localisation over a well defined crack surface.

In the third set of tests, a pseudo-3D (columnar) morphology subjected to the combined action of a progressive uniaxial strain load and a concentrated hydrogen source is analysed. The boundary conditions for this tests are represented in Fig. $7 \mathrm{7a}$, where the boundary region over which the external hydrogen concentration acts is highlighted; also in this case the external concentration is 


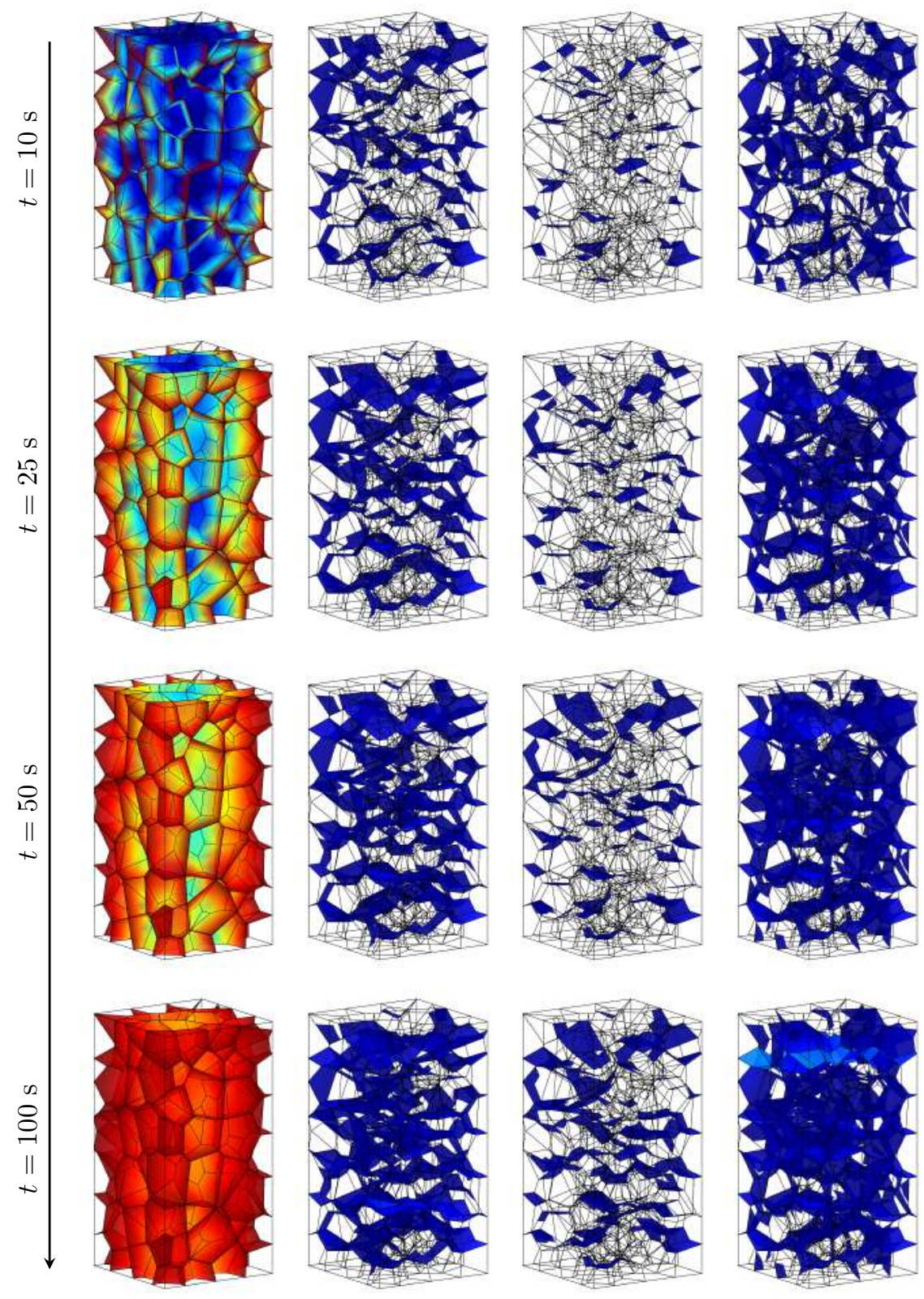

(a)

(b)

(c)

(d)

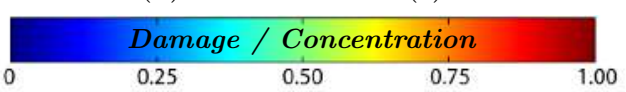

Figure 4: Contour plots for the stress relaxation tests: a) Hydrogen relative concentration evolution: $D=1.0 \mu \mathrm{m}^{2} / \mathrm{s}$; b) Damage evolution: $\left.\lambda_{0}=4.0 \cdot 10^{-2} \mu \mathrm{m}, \gamma=0.5 ; c\right)$ Damage evolution: $\left.\lambda_{0}=3.0 \cdot 10^{-2} \mu \mathrm{m}, \gamma=0.5 ; d\right)$ Damage evolution: $\lambda_{0}=3.0 \cdot 10^{-2} \mu \mathrm{m}, \gamma=0.9$. 


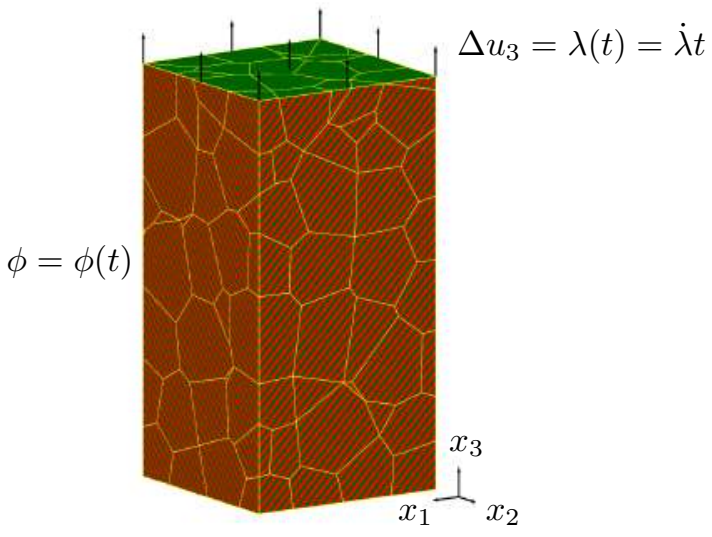

(a)

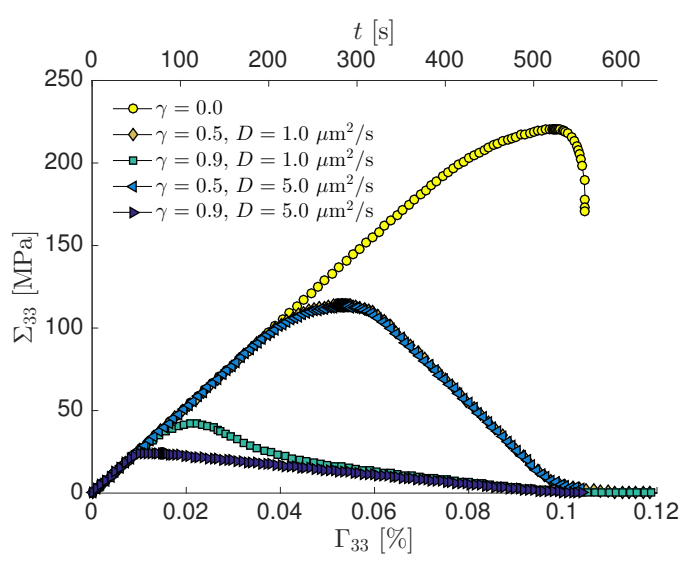

(c) $\dot{\lambda}=1.05 \cdot 10^{-4} \mu \mathrm{m} / \mathrm{s}$

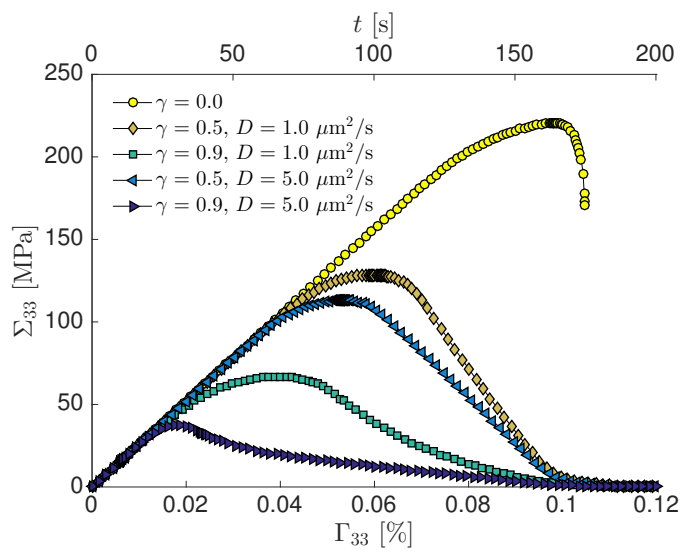

(b) $\dot{\lambda}=3.36 \cdot 10^{-4} \mu \mathrm{m} / \mathrm{s}$

Figure 5: Progressive loading tests under the action of external hydrogen environment: $a$ ) Schematic representation of the boundary conditions for the 200-grain morphology subject to prescribed progressive uniaxial strain and exposed to the aggressive species over the four lateral faces. Macro stress-strain curves for $b) \dot{\lambda}=3.36 \cdot 10^{-4} \mu \mathrm{m} / \mathrm{s}$ and $c$ ) $\dot{\lambda}=1.05 \cdot 10^{-4} \mu \mathrm{m} / \mathrm{s}$. $d$ ) Effect of different loading rates $\dot{\lambda}$ on the macro stress-strain curves. 


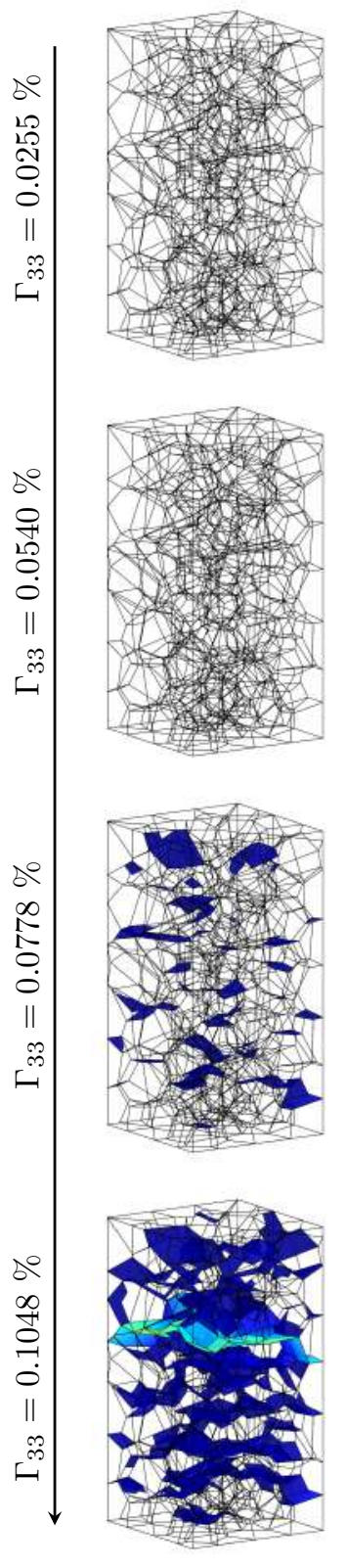

(a)
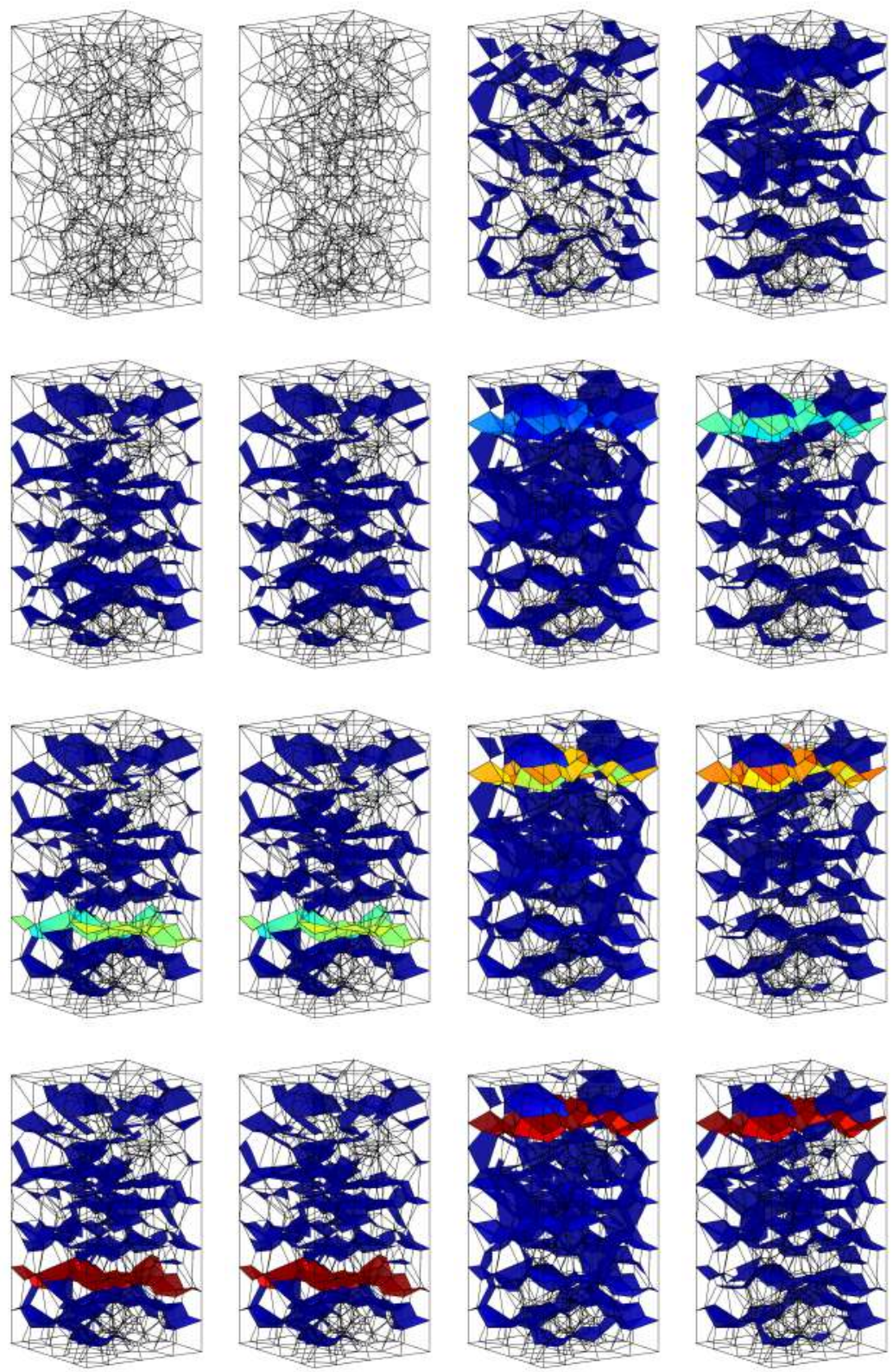

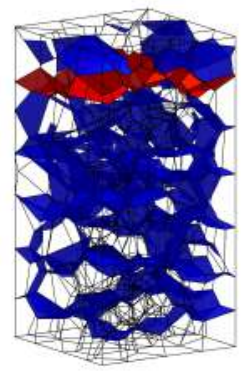

(d)

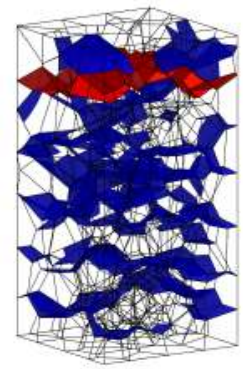

(e) (c)

(b)

\begin{tabular}{rrr}
\multicolumn{1}{c|}{ Damage } & & \\
0.25 & 0.50 & 0.75
\end{tabular}

Figure 6: Damage evolution contour plots for the progressive loading tests with hydrogen environment acting over the four lateral faces of the aggregate: $a$ ) no hydrogen-related interface degradation, i.e. $\gamma=0 ; b) \gamma=0.5, D=5.0$ $\left.\left.\mu \mathrm{m}^{2} / \mathrm{s}, \dot{\lambda}=3.36 \cdot 10^{-4} \mu \mathrm{m} / \mathrm{s} ; c\right) \gamma=0.5, D=5.0 \mu \mathrm{m}^{2} / \mathrm{s}, \dot{\lambda}=1.05 \cdot 10^{-4} \mu \mathrm{m} / \mathrm{s} ; d\right) \gamma=0.9, D=1.0 \mu \mathrm{m}^{2} / \mathrm{s}$, $\dot{\lambda}=3.36 \cdot 10^{-4} \mu \mathrm{m} / \mathrm{s} ;$ e) $\gamma=0.9, D=1.0 \mu \mathrm{m}^{2} / \mathrm{s}, \dot{\lambda}=1.85 \cdot 10^{-4} \mu \mathrm{m} / \mathrm{s}$. 


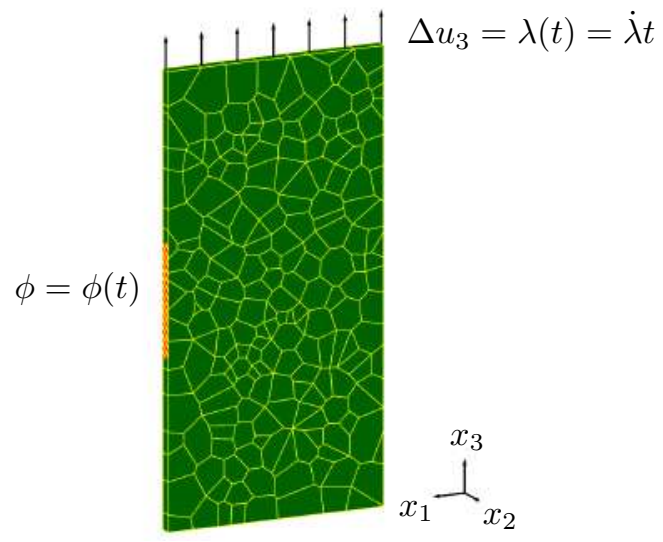

(a)

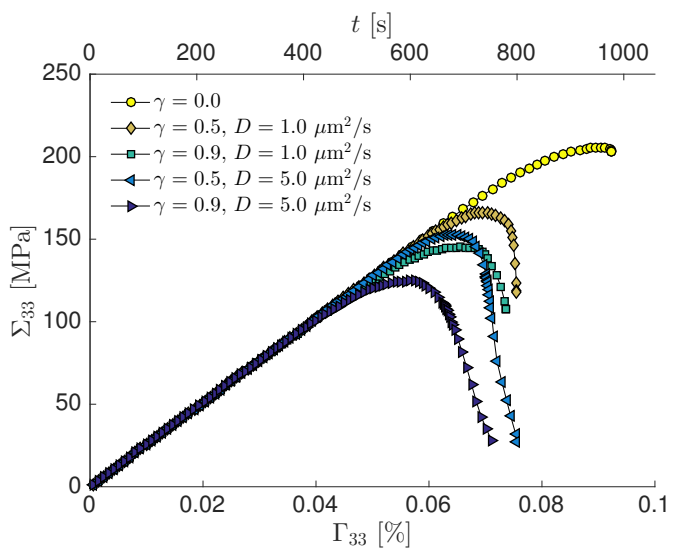

(b)

Figure 7: Tests for uniaxial strain progressive loading of a 200-grain pseudo-3D morphology exposed to a localised external source of hydrogen: $a$ ) Schematic representation of the boundary conditions; $b$ ) Macro stress-strain curves for different values of $\gamma$ and $D$.

smoothly increased from zero to the saturation value.

The purpose of this set of tests is the investigation of cases of specific susceptible grain boundaries or of localised environmental action due to the damage or reduced performance of a coating layer.

Fig.(7b) shows different macro stress-strain curves at a fixed loading rate for different combinations of effective diffusivity $D$ and degradation parameter $\lambda$. The same considerations as those valid in the previously analysed fully 3D test apply for the macro-curves, in terms of the overall effect of $D$ and $\gamma$ on the features of the diffusion/degradation process.

On the other hand, the contour plots shown in Fig. (8) highlight how the presence of a concentrated diffusion source results in a clearly identifiable damage and crack path; in particular the figure renders the evolution of the hydrogen concentration for a fixed value of $D$ and the damage levels and crack evolution for two different values of $\gamma$ at different times.

Fig.(9) shows the final crack path for a pseudo-3D morphology in the cases of no hydrogen diffusion and of a concentrated hydrogen diffusion source, with different combinations of $D$ and $\gamma$. It is highlighted again how in the presence of concentrated diffusion the damage process localises in a well defined crack path.

In the last test, the fully 3D counterpart of the previous case is analysed, with a fully $3 \mathrm{D}$ morphology subjected to a progressive tensile uniaxial strain and a localised environmental agent. 


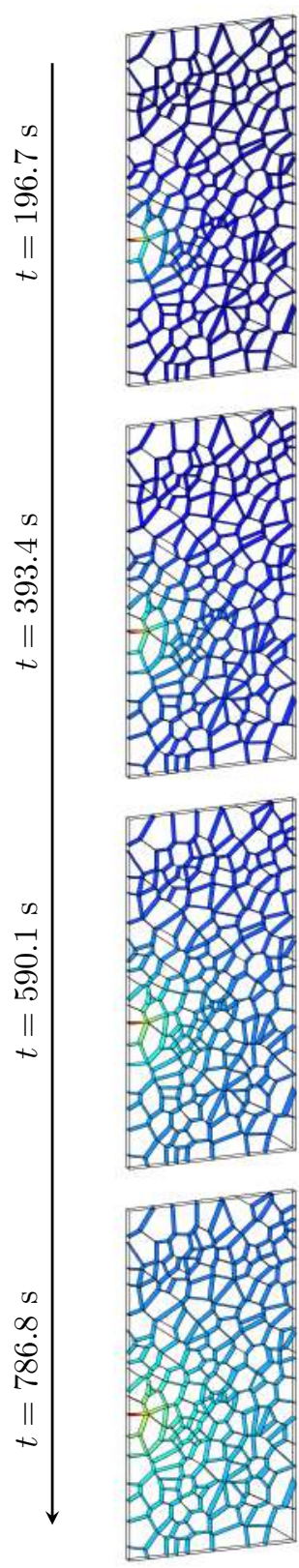

(a)
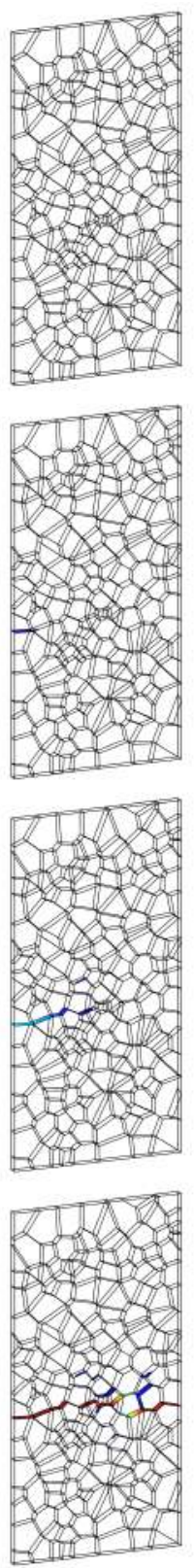

(b)
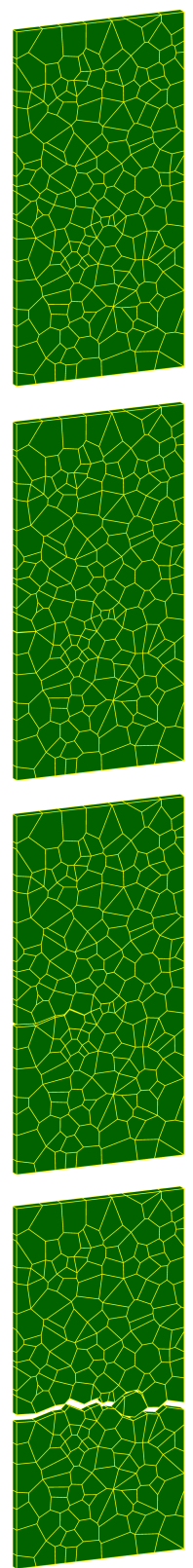

(c)
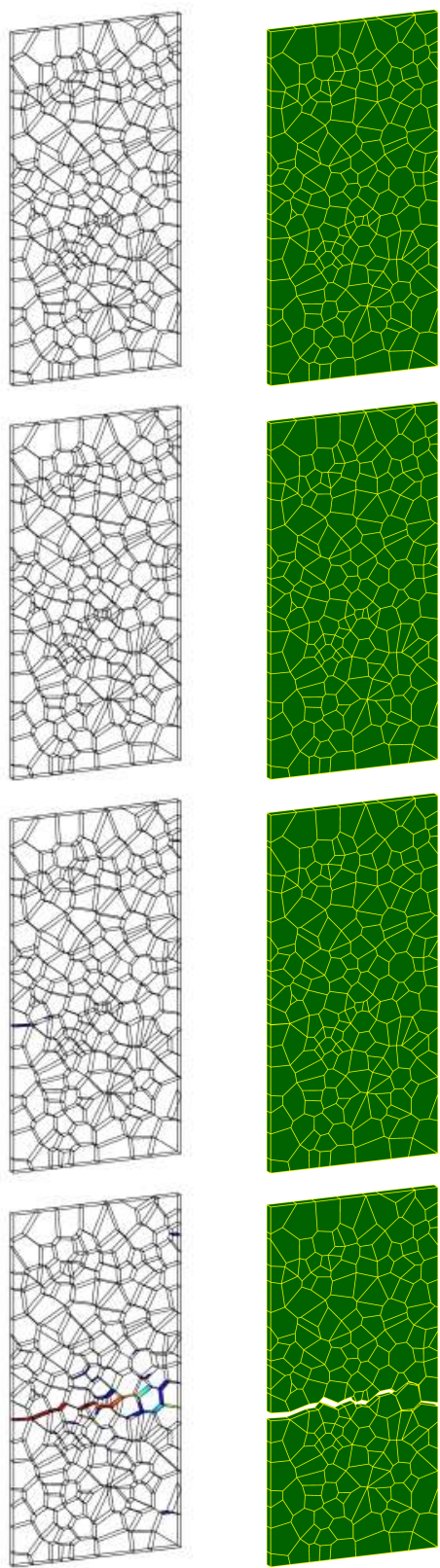

(d)

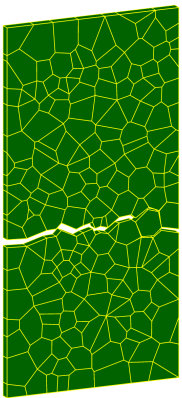

(e)

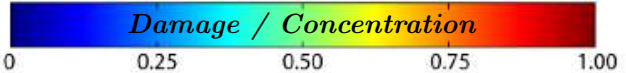

Figure 8: Contour plots for uniaxial strain progressive loading of a 200-grain pseudo-3D morphology exposed to a localised external source of hydrogen (loading rate $\dot{\lambda}=0.75 \cdot 10^{-4} \mu \mathrm{m} / \mathrm{s}$ ). a) Relative hydrogen concentration evolution: $\left.D=5.0 \mu \mathrm{m}^{2} / \mathrm{s} ; b\right)$ Damage evolution: $\left.\gamma=0.9 ; c\right)$ Crack path: $\gamma=0.9 ; d$ ) Damage evolution: $\left.\gamma=0.5 ; e\right)$ Crack path: $\gamma=0.5$. 


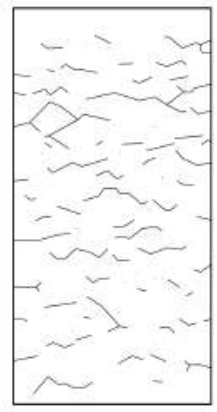

(a)

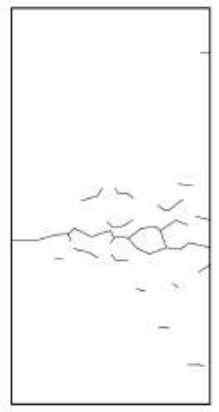

(b)

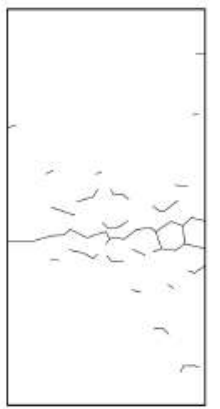

(c)

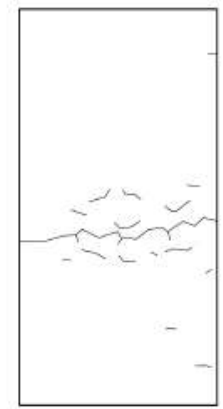

(d)

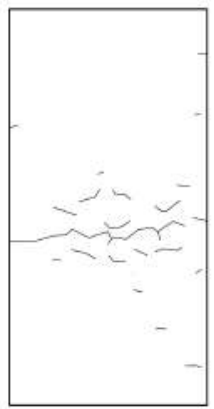

(e)

Figure 9: Developed crack paths for the progressive loading tests with localised hydrogen environment $\left(\dot{\lambda}=0.75 \cdot 10^{-4}\right.$ $\mu \mathrm{m} / \mathrm{s}): a)$ no hydrogen-related interface degradation, i.e. $\left.\gamma=0 ; b) \gamma=0.9, D=5.0 \mu \mathrm{m}^{2} / \mathrm{s} ; c\right) \gamma=0.5, D=5.0$ $\left.\left.\mu \mathrm{m}^{2} / \mathrm{s} ; d\right) \gamma=0.9, D=1.0 \mu \mathrm{m}^{2} / \mathrm{s} ; e\right) \gamma=0.5, D=1.0 \mu \mathrm{m}^{2} / \mathrm{s}$.

Fig. (10a schematises the boundary conditions with the localised hydrogen source on one of the external faces. The macro stress-strain curves, for different combinations of the diffusive/degradation parameters, are shown in Fig. $10 \mathrm{~b}$.

Fig.(11) shows the contour plots, at different times, of the hydrogen concentration associated with different values of $D$; also shown are the damage levels associated with the two tested values of diffusion, compared with the damage evolution induced by the purely mechanical loading, without environmental degradation. Also in the present tests, the localised environmental aggressive action results in the localisation of damage in the microstructure, with a clearly identifiable 3D intergranular crack path.

In concluding this section, it may be relevant to give some additional considerations about the applied boundary conditions. In the above tests, displacement boundary conditions have been enforced. In particular, constant displacements (and then macro-strains) have been applied to simulate the relaxation of polycrystalline specimens in aggressive environment. On the other hand, it could be of interest to study the creep of specimens subjected to corrosion, measuring the evolution of macro-strains under the action of constant external loads, applied for example through constant boundary tractions. Being the primary variables of the formulation both boundary displacements and tractions, the proposed technique can naturally handle constant or variable traction boundary conditions, allowing to follow the specimen creep up to the failure, as shown for example in 


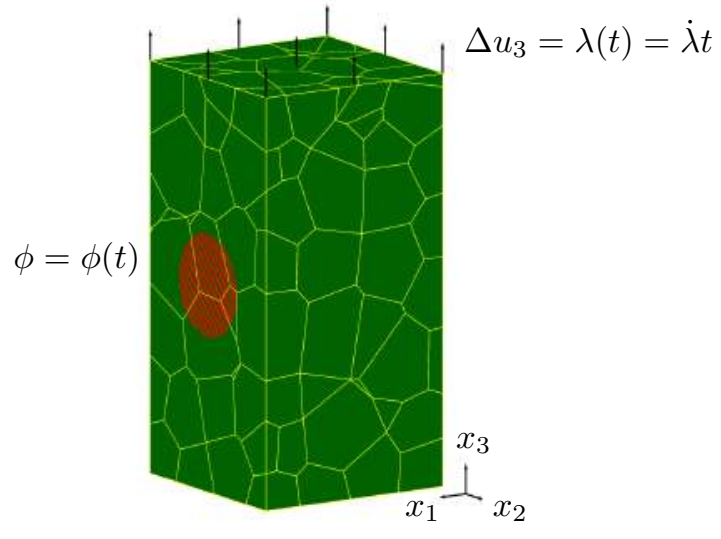

(a)

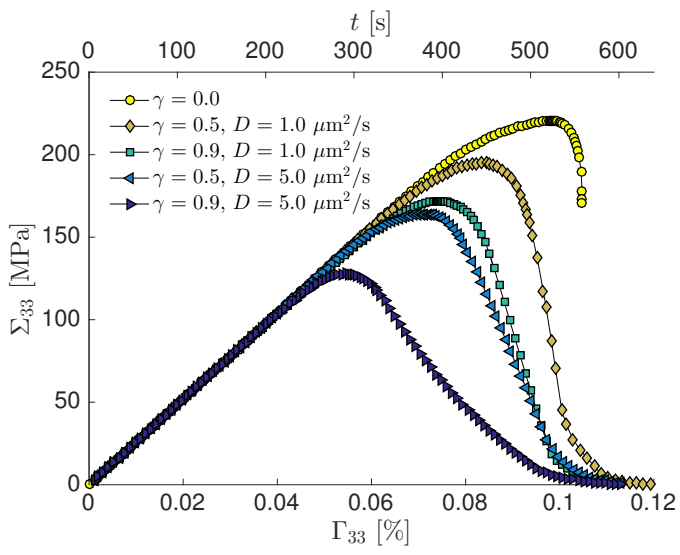

(b)

Figure 10: Tests for uniaxial strain progressive loading of a 200-grain fully 3D morphology exposed to a localised external source of hydrogen: a) Schematic representation of the boundary conditions; $b$ ) Macro stress-strain curves for different values of $\gamma$ and $D$.

Ref. 39, in which grain-boundary cavitation in polycrystalline materials has been modelled within the present framework.

\section{Discussion and future research}

The developed framework offers the advantage of a simplified computational representation of the aggregate, by virtue of the boundary integral formulation of the polycrystalline mechanics, which expresses the problem in terms in intergranular variables only, i.e. displacements and tractions.

The model is suitable for aggregates that mainly experience environmentally induced intergranular degradation under the effect of the external environment; the individual grains are in fact represented as anisotropic elastic domains not undergoing any bulk transformation or degradation. In other words, the diffusion of environmental agents, the subsequent degradation and the nucleation and evolution of irreversible damage under local effective tractions all take place at the intergranular interfaces, modelled through cohesive traction-separation laws embodying concentration and damage related parameters.

On the other hand, the choice of modelling the intergranular diffusion process through a finite element formulation, together with the lack of specific restrictions on the choice of the specific form 

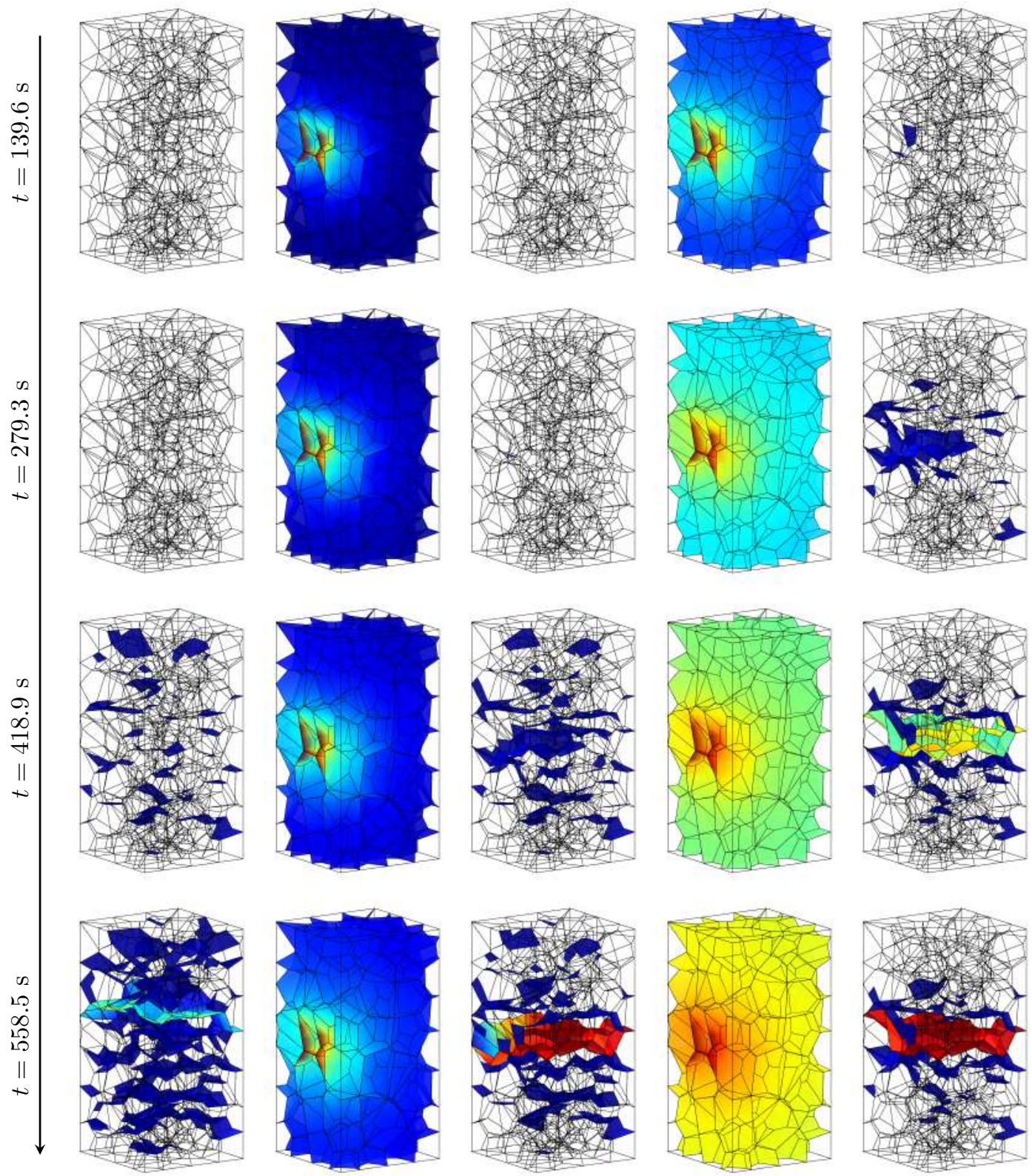

(a)

(b)

(c)

(d)

(e)

\begin{tabular}{ccccc|}
\hline & \multicolumn{4}{c|}{ Damage / Concentration } \\
\hline 0 & 0.25 & 0.50 & 0.75 & 1.00
\end{tabular}

Figure 11: Contour plots for uniaxial strain progressive loading of a 200-grain fully 3D morphology exposed to a localised external source of hydrogen (loading rate $\dot{\lambda}=1.05 \cdot 10^{-4} \mu \mathrm{m} / \mathrm{s}$ ). a) no hydrogen-related interface degradation, i.e. $\gamma=0 ; b)$ Hydrogen relative concentration evolution: $D=1.0 \mu \mathrm{m}^{2} / \mathrm{s} ; c$ ) Damage evolution: $\left.\gamma=0.5, D=1.0 \mu \mathrm{m}^{2} / \mathrm{s} ; d\right)$ Hydrogen relative concentration evolution: $D=5.0 \mu \mathrm{m}^{2} / \mathrm{s} ;$ e) Damage evolution: $\gamma=0.9, D=5.0 \mu \mathrm{m}^{2} / \mathrm{s}$. 
of the diffusion equation and the cohesive laws, except the requirement of physical consistence, ensures a certain generality in terms of representation of the behaviour of the aggregate interfaces.

In this respect, the framework could incorporate more sophisticated diffusion models [29, overcoming for example the Fourier paradox. On the other hand, the selection of the traction-separation tion, is by no means constrained in the present model: general cohesive laws can be selected and interesting developments could in fact be related to the formulation of functional forms derived from lower scale analyses, e.g. atomistic simulations addressed at resolving the interface decohesion process [11, 40]. proach suggested by Bomidi et al. [41, within the scope of continuum damage mechanics. However, the most critical aspect in this sense would be the formulation of suitable laws expressing the material degradation due to the simultaneous effect of both the cyclic loads and the aggressive environment, which cannot generally be expressed as a simple superposition [42]. The method could also be formulated in a dynamic boundary element framework, either in time or Laplace domain [43, 44, although the likely demanding computational requirements would necessitate suitable acceleration [45, 46] to render the analysis amenable.

Another aspect worth investigating could be the simulation of the penetration of the hydrogenrich environment in the intergranular space between loosening grains, at interfaces experiencing damage, opening and failure and simultaneously exposed to the aggressive environment. Opening grain boundaries could in fact be expected to exhibit absorption features intermediate between the free surface and the bulk values. This aspect has been addressed in the literature by employing mixed Dirichlet-Neumann boundary conditions [47. In the present formulation, the penetration of the aggressive environment could be simulated by making the effective diffusivity dependent on the local value of damage and opening and forcing the relative concentration at the exposed intergranular crack flanks at the saturation concentration levels. Moreover a macroscopic consistence flag could be used to avoid artifacts, e.g. at isolated failing interfaces located within the specimen but not yet possibly reached by the aggressive agents. These aspects could certainly be accommodated within the presented framework; however, as discussed in Ref.[19], the conditions prevailing within failing interfaces are complex and not perfectly understood, especially with respect to the chemical interaction between the newly formed surface and the aggressive environment. For these very 
reasons, this could be an intriguing research direction.

An interesting application of the framework could involve the study of specific materials in specific environments, with a more realistic representation of the interface properties, for example a distinction between susceptible and resistant intergranular interfaces or the representation of intergranular precipitates and/or inclusions. In other words, the inclusion of additional and more realistic intergranular physics, to be included in the model in agreement with available statistical information, and the study of specific materials in specific environments (known to experience intergranular degradation) would constitute an interesting research development.

Further developments could involve the inclusion of more complex constitutive behaviours for the bulk grains, e.g. crystal plasticity [48, 49] or trans-granular cracking ([50]), coupled with intragranular diffusion [17.

Another interesting research direction could be the inclusion of the developed scheme into a multi-scale computational framework [51, 52, which would allow linking the nucleation and evolution of material damage at the grain-scale with the failure of engineering components under the action of aggressive environments.

\section{Conclusions}

In this study a novel computational model for hydrogen assisted intergranular stress corrosion cracking in polycrystalline microstructures has been proposed, developed, implemented and tested.

The developed framework is based on the use of a hybrid BEM-FEM computational strategy: a boundary element formulation is employed to represent the mechanics of the crystal aggregate, whereas a finite element implementation is used to capture the intergranular diffusion of hydrogen, whose interfacial concentration evolves according to the Fick's second law. The link between the polycrystalline mechanics and the diffusion process, as well as between the two methods, is provided by intergranular cohesive interfaces, whose mechanical strength is degraded by the concentration of the diffused species.

The performed parametric analyses confirm the computational robustness of the developed framework and provide a qualitatively sound representation of the hydrogen assisted intergranular stress corrosion cracking. The developed formulation and implemented framework may constitute a valuable tool in the study of polycrystalline materials and components subjected to aggressive environments. 


\section{Acknowledgements}

The authors gratefully acknowledge CINECA's Italian Centre for Super Computing Applications and Innovation (SCAI) for granting access to their HPC infrastructure (http://www . hpc. cineca.it).

\section{Appendix A. Anisotropic Green's functions}

The kernels $U_{i j}(\boldsymbol{\xi}, \boldsymbol{\eta})$ and $T_{i j}(\boldsymbol{\xi}, \boldsymbol{\eta})$ appearing in Eqs.(1) are computed as

$$
U_{i j}(\boldsymbol{\xi}, \boldsymbol{\eta})=G_{i j}(\boldsymbol{\xi}, \boldsymbol{\eta}), \quad T_{i j}(\boldsymbol{\xi}, \boldsymbol{\eta})=n_{k}(\boldsymbol{\eta}) c_{j k p q} \frac{\partial G_{i p}}{\partial \eta_{q}}(\boldsymbol{\xi}, \boldsymbol{\eta})
$$

where $\boldsymbol{n}(\boldsymbol{\eta})=\left\{n_{i}(\boldsymbol{\eta})\right\}$ is the unit outward vector normal to the boundary at the point $\boldsymbol{\eta}$ and $G_{i j}(\boldsymbol{\xi}, \boldsymbol{\eta})$ are the 3D Green's functions for anisotropic elasticity, which are obtained as the solution of partial differential equations

$$
c_{i k j l} \frac{\partial^{2} G_{p j}}{\partial \eta_{k} \partial \eta_{l}}(\boldsymbol{\xi}, \boldsymbol{\eta})+\delta_{p i} \delta(\boldsymbol{\eta}-\boldsymbol{\xi})=0
$$

where $\boldsymbol{\xi}$ and $\boldsymbol{\eta}$ are the collocation and observation points, respectively, $c_{i j k l}$ is the anisotropic fourth-order elasticity tensor, $\delta_{p i}$ is the Kronecker delta and $\delta(\boldsymbol{\eta}-\boldsymbol{\xi})$ is the Dirac delta function. By applying the Fourier transform with respect to the variable $\boldsymbol{\eta}$ to Eq. A.2. and following the approach proposed in Ref. [27, the Green's functions $G_{i j}(\boldsymbol{\xi}, \boldsymbol{\eta})$ and their derivatives can be obtained in terms of spherical harmonics as follows

$$
\frac{\partial^{(I)} G_{i j}}{\partial r_{1}^{\left(\alpha_{1}\right)} \partial r_{2}^{\left(\alpha_{2}\right)} \partial r_{3}^{\left(\alpha_{3}\right)}}(\boldsymbol{r})=\frac{1}{4 \pi r^{I+1}} \sum_{\ell \in \mathcal{L}}^{\infty} P_{\ell}^{I}(0) \sum_{m=-\ell}^{\ell} \widetilde{G}_{i j,\left(\alpha_{1}, \alpha_{2}, \alpha_{3}\right)}^{\ell, m} Y_{\ell}^{m}(\hat{\boldsymbol{r}}),
$$

where $\boldsymbol{r} \equiv \boldsymbol{\eta}-\boldsymbol{\xi}, r=\sqrt{r_{k} r_{k}}, \hat{\boldsymbol{r}}=\boldsymbol{r} / r ; I=\alpha_{1}+\alpha_{2}+\alpha_{3}$ denotes the order of derivation and $\mathcal{L}$ is the set of positive even (odd) integers when $I$ is even (odd). $P_{\ell}^{I}(0)$ is the $\ell$-th associated Legendre polynomials of degree $I$ evaluated at 0 and $Y_{\ell}^{m}(\hat{\boldsymbol{r}})$ is the spherical harmonic of order $\ell$ and degree $m$. The coefficients $\widetilde{G}_{i j,\left(\alpha_{1}, \alpha_{2}, \alpha_{3}\right)}^{\ell, m}$ of the series are computed by means of the following integral over the unit sphere $S_{1}$ :

$$
\widetilde{G}_{i j,\left(\alpha_{1}, \alpha_{2}, \alpha_{3}\right)}^{\ell, m}=\int_{S_{1}}\left(\hat{\xi}_{1}\right)^{\alpha_{1}}\left(\hat{\xi}_{2}\right)^{\alpha_{2}}\left(\hat{\xi}_{3}\right)^{\alpha_{3}} \widetilde{G}_{i j}(\hat{\boldsymbol{\xi}}) \bar{Y}_{\ell}^{m}(\hat{\boldsymbol{\xi}}) \mathrm{d} S(\hat{\boldsymbol{\xi}}),
$$

being $\widetilde{G}_{i j}(\boldsymbol{\xi})=\left[c_{i k j l} \xi_{k} \xi_{l}\right]^{-1}$ and $\bar{Y}_{\ell}^{m}$ the complex conjugate of $Y_{\ell}^{m}$. The interested readers are referred to Ref. 27] for further details about the spherical harmonics expansions of the fundamental solutions. 


\section{References}

${ }_{425}^{4}$ [1] I. Scheider, M. Pfuff, W. Dietzel, Simulation of hydrogen assisted stress corrosion cracking

1. using the cohesive model, Engineering Fracture Mechanics 75 (15) (2008) 4283 - 4291. doi: http://dx.doi.org/10.1016/j.engfracmech.2007.10.002.

URL http://www.sciencedirect.com/science/article/pii/S0013794407003724

[2] N. Raykar, S. Maiti, R. S. Raman, Modelling of mode-i stable crack growth under hydrogen assisted stress corrosion cracking, Engineering Fracture Mechanics 78 (18) (2011) 3153 - 3165. doi:http://dx.doi.org/10.1016/j.engfracmech.2011.07.013. URL http://www.sciencedirect.com/science/article/pii/S0013794411002839

[3] N. Raykar, S. Maiti, R. S. Raman, S. Aryan, Study of hydrogen concentration dependent growth of external annular crack in round tensile specimen using cohesive zone model, Engineering Fracture Mechanics 106 (2013) 49 - 66. doi:http://dx.doi.org/10.1016/j. engfracmech.2013.04.007.

URL http://www .sciencedirect.com/science/article/pii/S0013794413001665

[4] C. Leas, E. Hondros, Intergranular microchemistry and stress corrosion cracking, Proceedings of the Royal Society of London. A. Mathematical and Physical Sciences 377 (1771) (1981) $477-501$.

[5] W. Wei, H. Grabke, The effect of alloying elements on the grain boundary segregation of phosphorus in iron and the intergranular corrosion of the Fe-P system, Corrosion Science 26 (3) (1986) $223-236$.

[6] S. Lynch, Hydrogen embrittlement phenomena and mechanisms, Corros Rev 30 (2012) 105123.

[7] J. Song, W. Curtin, Atomic mechanism and prediction of hydrogen embrittlement in iron, Nature materials 12 (2) (2013) 145-151.

[8] S. Wang, M. Martin, P. Sofronis, S. Ohnuki, N. Hashimoto, I. Robertson, Hydrogen-induced intergranular failure of iron, Acta Materialia 69 (0) (2014) 275 - 282. 
[15] A. King, G. Johnson, D. Engelberg, W. Ludwig, J. Marrow, Observations of intergranular stress corrosion cracking in a grain-mapped polycrystal, Science 321 (5887) (2008)

[9] A. Van der Ven, G. Ceder, Impurity-induced van der waals transition during decohesion, Phys. Rev. B 67 (2003) 060101. doi:10.1103/PhysRevB.67.060101. URL https://link.aps.org/doi/10.1103/PhysRevB.67.060101

[10] D. Jiang, E. A. Carter, First principles assessment of ideal fracture energies of materials with mobile impurities: implications for hydrogen embrittlement of metals, Acta Materialia 52 (16) (2004) 4801 - 4807. doi:http://dx.doi.org/10.1016/j.actamat.2004.06.037 URL http://www.sciencedirect.com/science/article/pii/S1359645404003854

[11] S. Serebrinsky, E. Carter, M. Ortiz, A quantum-mechanically informed continuum model of hydrogen embrittlement, Journal of the Mechanics and Physics of Solids 52 (10) (2004) 24032430.

[12] I. Simonovski, L. Cizelj, Towards modeling intergranular stress corrosion cracks on grain size scales, Nuclear Engineering and Design 246 (2012) 107-114.

[13] I. Benedetti, F. Barbe, Modelling polycrystalline materials: an overview of three-dimensional grain-scale mechanical models, Journal of Multiscale Modelling 5 (01) (2013) 1350002.

[14] T. Marrow, L. Babout, A. Jivkov, P. Wood, D. Engelberg, N. Stevens, P. Withers, R. Newman, Three dimensional observations and modelling of intergranular stress corrosion cracking in austenitic stainless steel, Journal of Nuclear Materials 352 (13) (2006) 62-74.

382-385. arXiv:http://science.sciencemag.org/content/321/5887/382.full.pdf, doi: $10.1126 /$ science.1156211.

URL http://science.sciencemag.org/content/321/5887/382

[16] S. Rahimi, D. Engelberg, J. Duff, T. Marrow, In situ observation of intergranular crack nucleation in a grain boundary controlled austenitic stainless steel, Journal of Microscopy 233 (3) (2009) 423-431.

[17] A. Musienko, G. Cailletaud, Simulation of inter-and transgranular crack propagation in polycrystalline aggregates due to stress corrosion cracking, Acta materialia 57 (13) (2009) 38403855. doi:10.1016/j.actamat.2009.04.035. 
¡18] M. Kamaya, M. Itakura, Simulation for intergranular stress corrosion cracking based on a three-dimensional polycrystalline model, Engineering Fracture Mechanics 76 (3) (2009) 386 401. doi:http://dx.doi.org/10.1016/j.engfracmech.2008.11.004. URL//WwW.sciencedirect.com/science/article/pii/S0013794408003068

[19] J. Rimoli, M. Ortiz, A three-dimensional multiscale model of intergranular hydrogenassisted cracking, Philosophical Magazine 90 (21) (2010) 2939-2963. doi:10.1080/ 14786431003752134

[20] I. Benedetti, M. Aliabadi, A three-dimensional grain boundary formulation for microstructural modeling of polycrystalline materials, Computational Materials Science 67 (2013) 249 - 260. doi:http://dx.doi.org/10.1016/j.commatsci.2012.08.006 URL //www.sciencedirect.com/science/article/pii/S0927025612004958

[21] I. Benedetti, M. Aliabadi, A three-dimensional cohesive-frictional grain-boundary micromechanical model for intergranular degradation and failure in polycrystalline materials, Computer

1. Methods in Applied Mechanics and Engineering 265 (2013) 36-62. doi:10.1016/j.cma.2013. 05.023

[22] V. Gulizzi, A. Milazzo, I. Benedetti, An enhanced grain-boundary framework for computational homogenization and micro-cracking simulations of polycrystalline materials, Computational Mechanics 56 (4) (2015) 631-651. doi:10.1007/s00466-015-1192-8.

[23] C. H. Rycroft, Voro++: A three-dimensional Voronoi cell library in C++, Chaos 19 (2009) 041111.

[24] R. Quey, P. R. Dawson, F. Barbe, Large scale 3D random polycrystals for the finite element method: Generation, meshing and remeshing, Computer Methods in Applied Mechanics and Engineering 200 (2011) 1729-1745.

[25] P. Banerjee, The boundary element methods in engineering, McGraw-Hill, 1994, (pp. 177-188).

[26] M. H. Aliabadi, The boundary element method: applications in solids and structures., Vol. 2, John Wiley \& Sons Ltd, England, 2002. 
[27] V. Gulizzi, A. Milazzo, I. Benedetti, Fundamental solutions for general anisotropic multifield materials based on spherical harmonics expansions, International Journal of Solids and Structures 100 (2016) 169-186. doi:10.1016/j.ijsolstr.2016.08.014.

[28] G. Sfantos, M. Aliabadi, A boundary cohesive grain element formulation for modelling intergranular microfracture in polycrystalline brittle materials, International journal for numerical methods in engineering 69 (8) (2007) 1590-1626. doi:10.1002/nme.1831.

[29] J. N. Reddy, D. K. Gartling, The finite element method in heat transfer and fluid dynamics, CRC press, 2010.

[30] V. I. Arnold, Ordinary Differential Equations, Springer-Verlag Berlin Heidelberg, 1992.

[31] R. B. Sidje, Expokit: a software package for computing matrix exponentials, ACM Transactions on Mathematical Software (TOMS) 24 (1) (1998) 130-156.

[32] A. Kuzmin, M. Luisier, O. Schenk, Fast methods for computing selected elements of the greens function in massively parallel nanoelectronic device simulations, in: F. Wolf, B. Mohr, D. Mey (Eds.), Euro-Par 2013 Parallel Processing, Vol. 8097 of Lecture Notes in Computer Science, Springer Berlin Heidelberg, 2013, pp. 533-544.

[33] O. Schenk, M. Bollhöfer, R. A. Römer, On large-scale diagonalization techniques for the anderson model of localization, SIAM Rev. 50 (1) (2008) 91-112.

[34] O. Schenk, A. Wchter, M. Hagemann, Matching-based preprocessing algorithms to the solution of saddle-point problems in large-scale nonconvex interior-point optimization, Computational Optimization and Applications 36 (2-3) (2007) 321-341.

[35] Y. Liu, Fast multipole boundary element method: theory and applications in engineering, Cambridge university press, 2009.

[36] M. Bebendorf, Hierarchical matrices: a means to efficiently solve elliptic boundary value problems, Vol. 63, Springer Science \& Business Media, 2008.

[37] I. Benedetti, M. Aliabadi, G. Davì, A fast 3d dual boundary element method based on hierarchical matrices, International Journal of Solids and Structures 45 (7) (2008) 2355 - 2376. 
[38] I. Benedetti, A. Milazzo, M. H. Aliabadi, A fast dual boundary element method for 3d anisotropic crack problems, International Journal for Numerical Methods in Engineering 80 (10) (2009) 1356-1378. doi:10.1002/nme.2666

\section{URL http://dx.doi.org/10.1002/nme.2666}

[39] V. Gulizzi, A. Milazzo, I. Benedetti, A micro-mechanical model for grain-boundary cavitation in polycrystalline materials, in: Key Engineering Materials, Vol. 665, Trans Tech Publ, 2016, pp. 65-68.

[40] A. Tahir, R. Janisch, A. Hartmaier, Ab initio calculation of traction separation laws for a grain boundary in molybdenum with segregated c impurites, Modelling and Simulation in Materials Science and Engineering 21 (7) (2013) 075005.

[41] J. Bomidi, N. Weinzapfel, F. Sadeghi, Three-dimensional modelling of intergranular fatigue failure of fine grain polycrystalline metallic mems devices, Fatigue \& Fracture of Engineering Materials \& Structures 35 (11) (2012) 1007-1021.

[42] R. N. Parkins, B. S. Greenwell, The interface between corrosion fatigue and stress-corrosion cracking, Metal Science 11 (8-9) (1977) 405-413. arXiv:http://dx.doi.org/10.1179/msc. 1977.11.8-9.405, doi:10.1179/msc.1977.11.8-9.405.

URL http://dx.doi.org/10.1179/msc.1977.11.8-9.405

[43] P. Fedelinski, M. Aliabadi, D. Rooke, Boundary element formulations for the dynamic analysis of cracked structures, Engineering Analysis with Boundary Elements 17 (1) (1996) 45 - 56. doi:http://dx.doi.org/10.1016/0955-7997(95)00089-5. URL http://www.sciencedirect.com/science/article/pii/0955799795000895

[44] A. Galvis, P. Sollero, 2d analysis of intergranular dynamic crack propagation in polycrystalline materials a multiscale cohesive zone model and dual reciprocity boundary elements, Computers \& Structures 164 (2016) 1 -14. doi:http://dx.doi.org/10.1016/j.compstruc.2015.11. 004

URL http://www.sciencedirect.com/science/article/pii/S0045794915003016 
[45] I. Benedetti, M. Aliabadi, A fast hierarchical dual boundary element method for threedimensional elastodynamic crack problems, International Journal for Numerical Methods in

[46] A. Milazzo, I. Benedetti, M. Aliabadi, Hierarchical fast bem for anisotropic time-harmonic

[50] G. Geraci, M. Aliabadi, Micromechanical boundary element modelling of transgranular and intergranular cohesive cracking in polycrystalline materials, Engineering Fracture Mechanics 176 (2017) 351-374.

[51] G. Sfantos, M. Aliabadi, Multi-scale boundary element modelling of material degradation and fracture, Computer Methods in Applied Mechanics and Engineering 196 (7) (2007) 1310-1329. doi:10.1016/j.cma.2006.09.004.

[52] I. Benedetti, M. Aliabadi, Multiscale modeling of polycrystalline materials: A boundary element approach to material degradation and fracture, Computer Methods in Applied Mechanics 